\title{
Numerical Analysis of Boundary Layer Flow and Heat Transfer over a Stretching and Non-Stretching Bullet- Shaped Object
}

\author{
By Mohammed Ali ${ }^{*} \&$ Md. Abdul Alim ${ }^{ \pm}$
}

\begin{abstract}
The two-dimensional axisymmetric magnetohydrodynamic boundary layer flow with heat transfer of Newtonian fluid over a stretching and non-stretching bullet-shaped object has been investigated. Therefore, fluid flow and heat transfer have been investigated in two types of flow geometries such as the thicker surface $(s \geq 2)$ and the thinner surface $(\mathrm{O}<\mathrm{s}<2)$ of the bullet-shaped object. The present analysis also focuses on the physical relevance and accurate trends of the boundary layer profiles which are adequate in the laminar boundary layer flow. The novelty of this current work is to discuss the effect of shape and size (surface thickness parameter s) and the stretching factor of the bullet-shaped object on the fluid velocity and temperature profiles within the boundary layer region also develop the relationship between the dependent and independent parameters by the correlation coefficient. The partial differential equations of momentum and energy have been reduced to a system of nonlinear ordinary differential equations along with the transformed boundary conditions by applying the local similarity transformations. These coupled non-linear ordinary differential equations' governing the flow field has been solved by the Spectral QuasiLinearization Method (SQLM). The numerical analysis of the SQLM has been carried out with MATLAB for investigating the effect of various controlling parameters on the flow fields. The residual error infinity norms have been analyzed to determine the speed of convergence and accuracy of the method. The numerical results have been displayed graphically and in tabular form and the physical behavior of the problem also discussed. The investigation shows that in the case of a thicker bullet-shaped object $(s \geq 2)$ the velocity profile does not approach the ambient condition asymptotically but intersects the axis with a steep angle and the boundary layer structure has no definite shape whereas in the case of a thinner bullet-shaped object $(0<s<2)$ the velocity profile converge the ambient condition asymptotically and the boundary layer structure has a definite shape. It is also noticed that thinner bullet-shaped object acts as good cooling conductor compared to thicker bullet-shaped object and the wall friction can be reduced much when thinner bullet-shaped object $(0<s<2)$ is used rather than the thicker bullet-shaped object $(s \geq 2)$ in both types of non-stretching or stretching bullet-shaped object $(\varepsilon=$ or $>0)$.
\end{abstract}

Keywords: forced convection, correlation coefficient, multiple regression, MHD, stretching

\footnotetext{
*Associate Professor, Department of Mathematics, Chittagong University of Engineering \& Technology, Bangladesh.

${ }^{ \pm}$Professor, Department of Mathematics, Bangladesh University of Engineering \& Technology, Bangladesh.
} 


\section{Introduction}

A systematic and detailed review of studies done in the past related to the present problem is under study. This helps us to understand the problems with its historical background, current status, and scope of application. The review of literature provides a synthesis of many documents and articles. The main purpose of the review is to summarize the related fields of interest coming from different mediums. The review helps us that how important the topic is and summarized the article for all that relates to the topic is essential. When an external flow past along objects it encompasses a variety of fluid mechanics phenomena. The nature of the flow field depends on the shape of the object. Even the simplest shaped objects, like a plate, may produce rather complex flows. Therefore, the flow pattern and related forces depend strongly on various parameters such as size, orientation, speed, and fluid properties. Therefore, the present problem will be helpful for designing bullet-shaped objects like Rocket, Missile, Aero plane, Bullet Train and Submarine, etc.

Due to the high applicability of this problem in such industrial phenomena, a large number of theoretical investigations are observed with different effects of physical parameters that have been presented during the last decades. Abo-Eldahab and Salem (2004) studied the boundary layer flow of non-Newtonian fluid and energy transfer over a power-law stretching surface with heat flux and observed that thermal boundary layer thickness reduces for the increasing values of mixed convection parameter but the reverse trend arises due to viscous dissipation, as a result, the energy transfer enhances. Aftab et al. (2018) analyzed the boundary layer power-law fluid flow over a moving permeable flat plate with viscous dissipation and heat generation. Ahmad et al. (2002) studied the mixed convection boundary layer flow by using the SQLM. This study concluded that the SQLM is suitable than another method. Later Ahmad et al. (2001) performed Bellman QLM for Neumann problems. Ajala et al. (2019) analyzed the boundary layer flow and energy transfer by the effect of the magnetic field, variable viscosity, and thermal radiation. Ajaykumar and Srinivasa (2020) studied the effect of variable viscosity on unsteady Magneto Hydrodynamics (MHD) laminar boundary layer flow with heat transfer over a stretching surface. Alarifi et al. (2019) discussed the influence of the sink or source on MHD boundary layer flow with heat transfer over a vertical stretching surface. Asaithambi (1998) discussed the Falkner - Skan equation by applying the finite difference method and found a similar solution. Ashwini and Eswara (2012) discussed that the boundary layer separation is delayed due to magnetic field parameter and the dual solutions are arises in case of decelerating flow regime. Ashwini and Eswara (2015) performed the unsteady MHD decelerating boundary layer wedge flow with heat generation. Awaludin et al. (2018) developed the stability model of MHD boundary layer flow over a stretching wedge. Daba et al. (2015) studied the mixed convection BL flow and heat transfer with convective boundary conditions over a vertical stretching surface. Marneni and Ashraf (2015) observed that the momentum and thermal boundary layer thicknesses decrease with the increase of Eckert number in the presence of suction and heat absorption when the wedge stretches slower than the 
free stream flow, Ashwini and Eswara (2015) discussed that the dual solutions exist in forced convection decelerating flow regime and the magnetic field stabilizes the flow which in turn delays the boundary layer separation from the wedge surface, Kandasamy and Mohammad (2015) investigated theoretically the impact of a convective surface on the heat transfer characteristics of water-based nanofluids over a static or moving wedge in the presence of magnetic field with variable stream condition and show that the temperature distribution in a nanofluid in the presence of thermal radiation with magnetic influenced by the convection parameter. Alam et al. (2016) studied numerically the effects of variable fluid properties and thermophoresis on unsteady forced convective boundary layer flow along with a permeable stretching/shrinking wedge. The results show that the Prandtl number, as well as the Schmidt number, varies significantly within the boundary layer for the flow with variable thermal conductivity and viscosity, Falana et al. (2016) studied the influence of Brownian motion and thermophoresis on a nonlinearly permeable stretching sheet in a nanofluid and found that the temperature rises for Brownian motion, thermophoresis and stretching ratio, Mabood et al. (2016) has been carried out to examine the effects of volume fraction of nanoparticles, suction/injection, and convective heat and mass transfer parameters on MHD stagnation point flow of water-based nanofluids and found that the friction factor and heat and mass transfer rates increase with magnetic field and suction/injection parameters. Nageeb et al. (2017) investigated the effects of thermal radiation, soret, and dufour parameters on mixed convection and nanofluid flow over a stretching sheet in the presence of a magnetic field. Mustaqim et al. (2018) studied the problem of the steady two-dimensional stagnation-point flow of heat and mass transfer over a shrinking sheet with the effect of radiation and velocity slip. Dual solutions are obtained. Therefore, they established a stability analysis that determines which solution is linearly stable and physically realizable. Kumar and Krishnan (2018) studied the axisymmetric boundary layer axial flow over a circular cylinder by using integral analysis. The analytical results are applicable to discuss the different flow regimes of axisymmetric boundary layers in the presence of pressure gradients. Aftab et al. (2018) investigated the combined effects of the thermal radiation, viscous dissipation, suction/injection, and internal heat generation/absorption on the boundary layer flow of a non-Newtonian powerlaw fluid over a semi-infinite permeable moving flat plate in parallel or reversely to a free stream. It was observed that the dual solutions exist when the flat plate and the free stream move in opposite directions. The velocity and temperature distributions are plotted and discussed for various values of the emerging physical parameters. Salleh et al. (2019) analyzed the steady boundary layer flow of a nanofluid past a thin needle under the influences of heat generation and chemical reaction. It is observed that the multiple (dual) solutions are likely to exist when the needle moves against the direction of the fluid flow. Therefore, the reduction in needle thickness provides the enlargement of the region of the dual solutions. The stable solution has been done by using a stability analysis. The obtained results indicate that the upper branch solutions are linearly stable, while the lower branch solutions are linearly unstable. The study also revealed that the rate of heat transfer is a decreasing function of heat generation parameter, while the rate of mass 
transfer is an increasing function of heat generation and chemical reaction parameters. Ibrahim and Tulu (2019) numerically inspected a steady laminar flow over a vertical stretching sheet with the existence of viscous dissipation, heat source/sink, and magnetic fields through a shooting scheme based Runge-KuttaFehlberg-integration algorithm. Findings reveal that the Nusselt number at the sheet surface augments, since the Hartmann number, stretching velocity ratio, and Hartmann number increase. Nevertheless, it reduces with respect to the heat generation/absorption coefficient. Jabeen et al. (2020) analyzed the MHD boundary layer flow over a nonlinear stretching sheet in a porous medium using semianalytical approaches and proved that the flow field is effectively appreciable by injection and suction. Megahed et al. (2021) studied the effects of the extended heat flux and variable fluid properties on the unsteady laminar MHD flow and heat transfer over a stretching sheet. Irfan et al. (2020) discussed the effects of unsteady MHD stagnation point flow of heat and mass transfer across a stretching/shrinking surface in a porous medium with internal heat generation/absorption, thermal radiation, and chemical reaction. Muthukumaran and Bathrinathan (2020) studied the analytical solution of mixed convection boundary layer flows with suction, injection (blowing) and viscous dissipation over a vertical stretching sheet near the stagnation point. Shateyi and Muzara (2020) discussed the boundary layer nanofluid flow over a Non-Linearly Stretching surface with the effects of viscous dissipation and chemical reaction.

The above discussion and review of the literature show that the mentioned works are restricted only for the case of flow around a flat plate, cone, wedge or cylindrical shape geometry with some physical conditions but to the best of author's knowledge, no published work has been found on boundary layer flow over a bullet-shaped geometry.

The main purpose of this problem is to investigate the MHD axisymmetric boundary layer flow and heat transfer over stretching and non-stretching bulletshaped object with internal heat generation parameter. Also analyzed the relationship between the physical parameters and flow profiles by applying the correlation coefficient and multiple regressions. Motivated by the previous studies, the present problem tries to fill the existing gaps in the field of boundary layer theory. The innovation of this present problem lies in the unification of more physical parameters into the governing equations and an attempt to give a thorough analysis of how the flow properties are affected by these parameters. Also, the novelty of the current paper lies in the application of the recently developed numerical method to solve these highly nonlinear equations.

So the purpose of the present work is to study the effect of various parameters such as magnetic effect, stretching factor, heat generation, size and shape of the surface on momentum and thermal behavior of the fluid, skin friction coefficient, rate of heat transfer, nature of solutions within the boundary layer over a bulletshaped object by using a spectral quasi-linearization iterative scheme. 


\section{Mathematical Model and Similarity Analysis}

Let us consider the steady two-dimensional MHD laminar boundary layer flow of an incompressible, electrically conducting, and viscous Newtonian fluid over a stretching bullet-shaped surface in a bulk fluid at a constant temperature $T_{\infty}$. A schematic representation of the physical model and coordinate system as shown in Figure 1. It is assumed that the free stream and the stretching velocities are $U_{\infty}$ and $U_{w}$ respectively, $\mathrm{x}$ is the coordinate measured along the bullet-shaped surface, $\mathrm{r}$ is the coordinate measured in the radial directions respectively.

Figure 1. Flow Geometry and Coordinate System

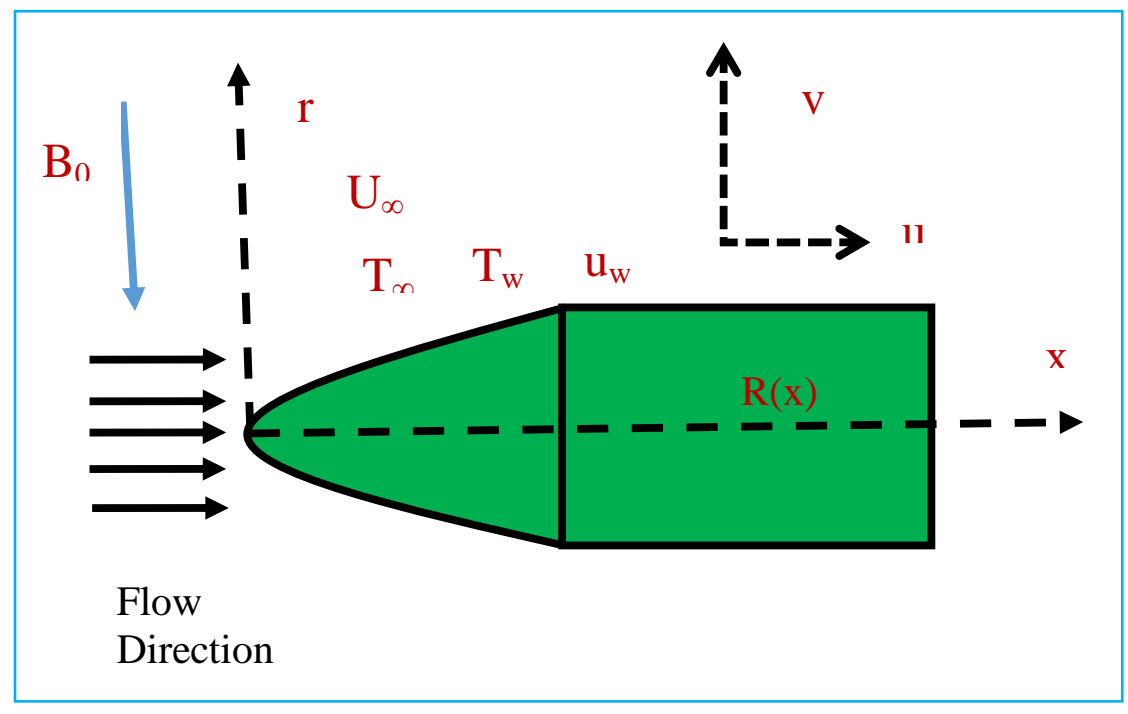

We assumed that the temperature of the surface is $T_{w}$, where $T_{w}>T_{\infty}$ corresponds to a heated surface (assisting flow) and $T_{w}<T_{\infty}$ corresponds to a cooled surface (opposing flow). The surface has an isothermal surface, and kept at temperatures $\mathrm{T}_{\mathrm{w}}$ such that the temperature differences within the flow are sufficiently small. The small magnitude of the temperature difference allows expression of the Taylor series expansion about the free stream temperature $\mathrm{T}_{\infty}$ as a linear function of temperature at any interior point within the flow region. The magnetic field $B_{0}$ is imposed perpendicular to the direction of the flow and the induced magnetic field can be neglected due to the smallest magnetic Reynolds number. The surface moves with a velocity $U_{w}(x)$, in the same or opposite direction of the free stream velocity $U_{\infty}(x)$. It is also assumed that the external electric field is zero and the electric field due to polarization of charges is negligible. The velocity of the surface $U_{w}(x)$, the free stream velocity $U_{\infty}(x)$ and the temperature of the surface $T_{w}$ are respectively defined as $U_{w}(x)=a x^{m}, U_{\infty}(x)=b x^{m}, T_{w}(x)=x^{2 m-1}$, where a and $\mathrm{b}(b>0)$ are the 
stretching rate of the sheet and straining rate parameters respectively with $a>0$ for stretching case and $a<0$ for the shrinking case. Also, $\mathrm{m}$ is the power-law index parameter of velocity and temperature. It may be noted that the constant $b$ is proportional to the free stream velocity far away from the surface. An axial pressure gradient is imposed inside the boundary layer due to variable axial freestream.

Therefore, the governing partial differential equations of continuity, momentum and thermal energy are as follows

Equation of continuity:

$$
\frac{\partial}{\partial x}(r u)+\frac{\partial}{\partial r}(r v)=0
$$

(1)

Momentum equation:

$$
\begin{aligned}
& \mathrm{u} \frac{\partial \mathrm{u}}{\partial \mathrm{x}}+\mathrm{v} \frac{\partial \mathrm{u}}{\partial r}=\mathrm{U} \frac{\mathrm{dU}}{\mathrm{dx}}+\frac{\mathrm{v}}{r}\left(\frac{\partial \mathrm{u}}{\partial \mathrm{r}}+r \frac{\partial^{2} \mathrm{u}}{\partial \mathrm{r}^{2}}\right)+\frac{\sigma \mathrm{B}_{0}{ }^{2}}{\rho}(\mathrm{U}-\mathrm{u}) \\
& \text { where }-\frac{1}{\rho} \frac{\partial p}{\partial x}=\mathrm{U}(x) \frac{\mathrm{dU}(x)}{\mathrm{dx}}
\end{aligned}
$$

(2)

Energy equation:

$\mathrm{u} \frac{\partial \mathrm{T}}{\partial \mathrm{x}}+\mathrm{v} \frac{\partial \mathrm{T}}{\partial \mathrm{r}}=\frac{\alpha}{r}\left(\frac{\partial T}{\partial r}+r \frac{\partial^{2} \mathrm{~T}}{\partial \mathrm{r}^{2}}\right)+\frac{Q}{\rho C_{p}}\left(T-T_{\infty}\right)$

The above equations are subject to the following boundary conditions

$$
\begin{gathered}
u=U_{w}(x), v=0, T=T_{w} \text { at } r=R(x) \text { and } \\
u \rightarrow U_{\infty}(x)=U, T \rightarrow T_{\infty} \text {, as } r \rightarrow \infty
\end{gathered}
$$

where $\mathrm{R}(\mathrm{x})$ prescribes the surface shape and size of the axisymmetric body, $\mathrm{u}$ and $\mathrm{v}$ are the velocity components along with the axial and radial directions $(x, r)$ respectively, $v$ is the kinematic viscosity of the fluid, $\rho$ is the density of the fluid, the term $\frac{\sigma B_{0}^{2}}{\rho} u$ describes the x component of the magnetic field where $\frac{\sigma B_{0}^{2}}{\rho}$ is the magnetic parameter which is the ratio of the electromagnetic force to the inertial force, $B_{0}$ is the magnetic field intensity, $\alpha$ is the thermal diffusivity of the fluid and $\mathrm{T}$ is the fluid temperature respectively. The magnetic field usually applied in the $\mathrm{y}$ direction normal to the boundary layer flow. The flow is in $\mathrm{x}$ direction whose velocity component is $\mathrm{u}$. Thus $\mathrm{J} \times \mathrm{B}$, representing Lorentz force becomes $-\frac{\sigma B_{0}^{2}}{\rho} u$. The negative sign in the term is because of retardation. The first term on the right-hand side of equation (2) refers to the pressure gradient in 
the stream wise direction, as calculated from the Euler's equation for inviscid fluid.

To obtain the similarity solutions for the system of equation (1) - equation (3) subject to the boundary conditions (4), we use the following axisymmetric similarity transformation as:

$$
\eta=\frac{U r^{2}}{v x}, \psi=v x f(\eta), T=x^{2 m-1} \theta(\eta)
$$

where $\psi$ is the stream function and is defined by the velocity components $u=\frac{1}{r} \frac{\partial \psi}{\partial r}$ and $v=-\frac{1}{r} \frac{\partial \psi}{\partial x}, \eta$ is the similarity variable, $U_{w}$ is the surface velocity and $U_{\infty}$ is the free stream velocity. The expression $\eta$ gives the shape and size of the body, when we put $\eta=\mathrm{s}$ in the expression $\eta=\frac{U r^{2}}{v x}$, we get the dimensionless radius $R(x)=\sqrt{\frac{v s x^{1-m}}{a+b}}$.

The continuity equation (1) is identically satisfied by the equation (5) and the equations (2) and (3) are transformed into non-dimensional, nonlinear and coupled ordinary differential equations by applying the similarity transformation as

$$
\begin{aligned}
& \eta f^{\prime \prime \prime}+f^{\prime \prime}+\frac{1}{2} f f^{\prime \prime}+\frac{m}{8}\left(1-4 f^{\prime 2}\right)+\frac{M}{8}\left(1-2 f^{\prime}\right)=0 \\
& \eta \theta^{\prime \prime}+\frac{1}{2} \theta^{\prime}+\frac{1}{2} \operatorname{Pr}\left[f \theta^{\prime}-(2 m-1) f^{\prime} \theta\right]+\frac{1}{4} \operatorname{Pr} Q^{*} \theta=0
\end{aligned}
$$

and the transformed boundary conditions are

$$
\begin{aligned}
& f(\eta)=0, f^{\prime}(\eta)=\frac{\varepsilon}{2}, \theta(\eta)=1, \text { at } \eta=s, \text { and } \\
& f^{\prime}(\eta) \rightarrow \frac{1}{2}, \theta(\eta) \rightarrow 0 \text { as } \eta \rightarrow \infty
\end{aligned}
$$

Where prime denotes differentiation with respect to $\eta, \varepsilon=\frac{U_{w}}{U_{\infty}}$ is the velocity ratio parameter between the surface and the composite velocity. It is mentioned here that the velocity ratio parameter $\varepsilon>1$ means that the surface stretches faster than that of the free stream flow and $\varepsilon<1$ means that the surface slower than that of the free stream flow. Here, $\varepsilon>0$ is for stretching and $\varepsilon<0$ is for shrinking. On the other hand, $\varepsilon=0$ and $\varepsilon=1$ correspond to a fixed surface in a moving fluid (Blasius flow) and a moving surface in a quiescent fluid (Sakiadis flow), respectively. The range $0<\varepsilon<1$ indicates that the surface and the fluid move in the same direction. If $\varepsilon<0$ the free stream is directed towards the positive $\mathrm{x}$ direction, while the surface moves towards the negative x-direction. When $\varepsilon>1$, the free stream is directed towards the negative $\mathrm{x}$-direction, while the surface moves towards the positive $\mathrm{x}$-direction. However, the present analysis has performed only for the case $\varepsilon \leq 2$. 
The dimensionless parameters $\mathrm{M}$ is the magnetic parameter, $\mathrm{m}$ is the powerlaw index parameter, $\varepsilon$ is the stretching parameter Pr is the Prandtl number, and $\mathrm{Q}^{*}$ is the heat generation parameter which is defined as

$$
\mathrm{M}=\frac{\sigma_{\mathrm{O}}^{2} \mathrm{x}}{2 \rho \mathrm{U}}, \varepsilon=\frac{\mathrm{U}_{w}}{\mathrm{U}}, \operatorname{Pr}=\frac{\nu}{\alpha}, Q^{*}=\frac{Q x}{\rho U_{w} c_{p}}
$$

In practical applications, two quantities of physical interest are to be determined, such as surface shear stress and the rate of heat transfer at the surface. These may be obtained in terms of the local skin-friction coefficient $C_{f}$ and the local Nusselt number, $N u_{x}$. The skin friction and Nusselt number are the dimensionless shear stress at the surface and dimensionless heat flux at the surface respectively and can be expressed as the following way.

We have the shear stress at the surface $\tau_{w}=\mu\left(\frac{\partial u}{\partial r}\right)_{r=0}$ and the local skin friction coefficient $C_{f}=\frac{2 \tau_{w}}{\rho U^{2}}$

By applying the equations (4) and (5), we obtain the local skin friction coefficient

$$
C_{f}=\frac{\mu}{\rho U^{2}}\left(\frac{\partial u}{\partial r}\right)_{r=0}=4 \sqrt{\frac{\eta}{\operatorname{Re}_{x}}} f^{\prime \prime}(\eta) \Rightarrow C_{f} \sqrt{\operatorname{Re}_{x}}=4 \sqrt{\eta} f^{\prime \prime}(\eta)
$$

The local Nusselt number for the convective boundary condition is defined as $N u_{x}=\frac{x q_{w}}{\kappa\left(T_{w}-T_{\infty}\right)}$. Where $q_{w}$ is the wall heat flux and can be defined as $q_{w}=-\kappa\left(\frac{\partial T}{\partial r}\right)_{r=0}$.

By using the equations (4) and (5), we obtain the local Nusselt number $N u_{x}\left(\operatorname{Re}_{x}\right)^{-1 / 2}=-2 \sqrt{\eta} \theta^{\prime}(\eta)$

\section{Procedure of Numerical Solution}

Bellman and Kalaba (1965) were the first to apply the QLM about half a century ago to solve nonlinear ordinary and partial differential equations. Since the differential equation is highly non-linear and it is almost impossible to find the closed-form analytic solution. The SQLM (2020) is a combination of two methods: (i) the Quasi-linearization method (QLM) and (ii) the Chebyshev spectral collocation method. The QLM is the generalization of Newton-Raphson-based method and is used to linearize the non-linear ODEs into linear ODEs. The QLM approaches that the difference between the approximate solution at the present iteration and the previous iteration is very small. The numerical simulation of the 
present problem is obtained with the help of SQLM which gives highly accurate results. Since the similarity variable converges to $\eta \rightarrow \infty$ but the present simulation has been performed for a finite domain of $\eta$. Therefore, the dimensionless velocity and energy distribution within the boundary layer asymptotically tends to a free stream velocity that satisfies the boundary conditions. The numerical solution for the above equations for various values of magnetic parameter $\mathrm{M}$, power-law index parameter $\mathrm{m}$, the thickness of the surface $\mathrm{s}$, stretching ratio $\varepsilon$ and Prandtl number $\operatorname{Pr}$ are analyzed using the quasi-linearization technique. Applying SQLM, the system of equations (6) and (7) are converted into the following iterative sequence of linear differential equations.

$$
\begin{gathered}
\mathrm{a}_{0, \mathrm{t}} f_{\mathrm{t}+1}^{\prime \prime \prime}(\eta)+\mathrm{a}_{1, \mathrm{t}} f_{\mathrm{t}+1}^{\prime \prime}(\eta)+\mathrm{a}_{2, \mathrm{t}} f_{\mathrm{t}+1}^{\prime}(\eta)+\mathrm{a}_{3, \mathrm{t}} f_{\mathrm{t}+1}(\eta)=R_{1, \mathrm{t}}(\eta) \\
\mathrm{b}_{0, \mathrm{t}} \theta_{t+1}^{\prime \prime}(\eta)+\mathrm{b}_{1, \mathrm{t}} \theta_{t+1}^{\prime}(\eta)+\mathrm{b}_{2, \mathrm{t}} \theta(\eta)+\mathrm{b}_{3, \mathrm{t}} f_{t+1}^{\prime}(\eta)+\mathrm{b}_{4, \mathrm{t}} f_{t+1}(\eta)=R_{2, t}(\eta)
\end{gathered}
$$

The subscripts $t$ and $t+1$ denote previous and current approximations.

The transformed boundary conditions are

$$
\begin{aligned}
& f_{t+1}\left(x N_{x}\right)=0, f_{t+1}\left(x N_{x-1}\right)=\frac{\varepsilon}{2}, \theta_{\mathrm{t}+1}\left(x N_{x}\right)=1, \text { at } \eta=0 \\
& f_{t+1}\left(x_{0}\right)=0, f_{t+1}\left(x_{0}\right)=\frac{1}{2}, \theta_{\mathrm{t}+1}\left(x_{0}\right)=0, \text { as } \eta \rightarrow \infty
\end{aligned}
$$

The variable coefficients obtained from the previous iteration are given by

$$
\begin{aligned}
& a_{0, t}=\frac{\partial f}{\partial f^{\prime \prime \prime}}=\eta, a_{1, t}=\frac{\partial f}{\partial f^{\prime \prime}}=\frac{1}{2}+\frac{1}{2} f_{t}, a_{2, t}=\frac{\partial f}{\partial f^{\prime}}=-m f_{t}^{\prime}-\frac{M}{4}, a_{3, t}=\frac{\partial f}{\partial f}=\frac{1}{2} f_{t}^{\prime \prime} \\
& b_{0, t}=\frac{\partial \theta}{\partial \theta^{\prime \prime}}=\eta, b_{1, t}=\frac{\partial \theta}{\partial \theta^{\prime}}=\frac{1}{2}+\frac{\operatorname{Pr}}{2} f_{t}, b_{2, t}=\frac{\partial \theta}{\partial \theta}=\frac{1}{4} \operatorname{Pr} Q^{*}-\frac{\operatorname{Pr}(2 m-1)}{2} f_{t}^{\prime} \\
& b_{3, t}=\frac{\partial \theta}{\partial f^{\prime}}=-\frac{\operatorname{Pr}(2 m-1)}{2} \theta_{t}, b_{4, t}=\frac{\partial \theta}{\partial f}=\frac{\operatorname{Pr}}{2} \theta_{t}^{\prime} \\
& R_{1, t}(\eta)=a_{0, t} f_{t}^{\prime \prime \prime}+a_{1, t} f_{t}^{\prime \prime}+a_{2, t} f_{t}^{\prime}+a_{3, t} f_{t}-F\left[\eta, f(\eta), f^{\prime}(\eta), f^{\prime \prime}(\eta) \ldots \ldots f^{n}(\eta)\right] \\
& =\frac{1}{2} f_{t} f^{\prime \prime}-\frac{m}{2} f_{t}^{2}-\frac{m}{8}-\frac{M}{8} \\
& R_{2, t}(\eta)=b_{0, t} \theta_{t}^{\prime \prime}+b_{1, t} \theta_{t}^{\prime}+b_{2, t} \theta_{t}+b_{3, t} f_{t}^{\prime}+b_{4, t} f_{t} \\
& -F\left[\eta, f(\eta), f^{\prime}(\eta), f^{\prime \prime}(\eta) \ldots . . f^{n}(\eta)\right]=\frac{\operatorname{Pr}}{2}\left[f_{t} \theta_{t}^{\prime}-(2 m-1) f_{t}^{\prime} \theta_{t}\right]
\end{aligned}
$$

The linear differential equations (9) and (10) are solved iteratively by using the Chebyshev spectral collocation method (CSCM). The linear transformation 
$x=\frac{2 \eta}{L_{\infty}}-1$ is used to convert the domain $[0, \infty)$ into the computational domain $[-1$, 1]. The unknown functions $f_{r+1}$ and $\theta_{r+1}$ are determined with the help of CSCM, and Chebyshev interpolating polynomials. The derivatives of the functions $f_{r+1}$ and $\theta_{r+1}$ at the Gauss-Lobatto collocation points are

$$
x_{i}=\cos \left(\frac{\pi i}{N}\right), i=0,1,2, \ldots . N \text { gives } \frac{d^{n} f_{t+1}\left(x_{i}\right)}{d x}=\sum_{k=0}^{N_{1}} D_{i k}^{n} f_{t+1}\left(x_{k}\right)=D^{n} F
$$

Where, $D=\frac{L_{\infty}}{2} \mathrm{k}, \mathrm{k}$ is $\left(\mathrm{N}_{1}+1\right) \times\left(\mathrm{N}_{1}+1\right)$ differentiation matrix and $F=\left[f_{t+1}\left(x_{0}\right), f_{t+1}\left(x_{1}\right), f_{t+1}\left(x_{2}\right), \ldots . f_{t+1}\left(x_{N-1}\right), f_{t+1}\left(x_{N}\right)\right]^{T}$. Similarly, the nth derivatives of $\theta_{t+1}$ are given by

$$
\frac{d^{n} \theta_{t+1}\left(x_{i}\right)}{d x}=\sum_{k=0}^{N_{1}} D_{i k}^{n} \theta_{t+1}\left(x_{k}\right)=D^{n} \Theta
$$

By applying the Equations (11) and (12), the Equations (9) and (10) yields

$$
\begin{aligned}
& {\left[\begin{array}{ll}
A_{11} & A_{12} \\
A_{21} & A_{22}
\end{array}\right]\left[\begin{array}{l}
F_{t+1} \\
\Theta_{t+1}
\end{array}\right]=\left[\begin{array}{l}
R_{1, t} \\
R_{2, t}
\end{array}\right], \text { Where } } \\
A_{11}= & a_{0, t} D^{3}+a_{1, t} D^{2}+a_{2, t} D+a_{3, t}, A_{12}=0 I, \\
A_{21}= & b_{3, t} D^{2}+b_{4, t}, A_{22}=b_{0, r} D^{2}+b_{1, t} D+b_{2, t}
\end{aligned}
$$

The matrix I is of order $\left(\mathrm{N}_{1}+1\right) \times\left(\mathrm{N}_{1}+1\right)$.

\section{Results and Discussion}

The non-linear ODEs of the present problem are solved by applying SQLM. The convergence criteria of the solution are performed by the use of solutionbased errors. These errors are defined by the differences between approximate solutions at the previous and current iteration levels $t$ and $t+1$, respectively. The error norms are defined as:

$$
\text { Error }_{f}=\max _{0 \leq i \leq N}\left\|F_{t+1, i}-F_{t, i}\right\| \text { and } \text { Error }_{\theta}=\max _{0 \leq i \leq N}\left\|\theta_{t+1, i}-\theta_{t, i}\right\|
$$

The infinity norms of the residual errors are defined by

$$
\|\operatorname{Re} s(f)\|_{\infty}=\left\|\eta f^{\prime \prime \prime}+f^{\prime \prime}+\frac{1}{2} f f^{\prime \prime}+\frac{m}{8}\left(1-4 f^{\prime 2}\right)+\frac{M}{8}\left(1-2 f^{\prime}\right)\right\| \text { and }
$$




$$
\|\operatorname{Re} s(\theta)\|_{\infty}=\| \eta \theta^{\prime \prime}+\frac{1}{2} \theta^{\prime}+\frac{1}{2} \operatorname{Pr}\left[\begin{array}{ll}
f & \left.\theta^{\prime}-(2 m-1) f^{\prime} \theta+\frac{1}{2} Q^{*} \theta\right] \|
\end{array}\right.
$$

The impact of physical parameters on velocity $f^{\prime}(\eta)$, temperature $\theta(\eta)$, skin friction, and the local Nusselt number is shown graphically. The numerical values of skin friction $\left(C_{f} \sqrt{\operatorname{Re}_{x}}\right)$ and Nusselt number $\left(N u_{x}\left(\sqrt{\operatorname{Re}_{x}}\right)^{-1}\right)$ which are equivalent to velocity gradient $f^{\prime \prime}(\eta)$ and temperature gradient $\theta^{\prime}(\eta)$ have been shown in Tables 1 and Table 2 respectively. The computations are done by taking $\mathrm{N}=50$ collocation points and solution-based errors are defined for the convergence of the numerical method. Again, Figures 16a and 16b show the error infinity norms and residual error infinity norms against iterations. Figure 16(a) represents the convergence for the present problem with iterations. From this, it is noticed that the error infinity norm decreases with the increasing number of iterations that confirm the convergence of the present method. So, it is observed that the present method converges after five iterations. On the other hand, Figure 16(b) assure the accuracy of the present method of less than $10^{-8}$ and $10^{-14}$ for $f(\eta)$ and $\theta(\eta)$ against after fourth iterations. It is seen that the residual error decreases with increasing the iterations. This proves the validity of the present method. The errors show that the SQLM is accurate giving errors of less than $10^{-8}$ within the fourth iteration. In this chapter the default parameters are taken as $M=$ 1.0, $m=1.0, \operatorname{Pr}=0.71, \epsilon=0.0,2.0$ and $s=0.05,2.0$ throughout the calculation. The fluid velocity profile and skin friction have been depicted graphically versus boundary layer co-ordinate $(\eta)$ in Figures 2-6 for the increasing effect of the controlling parameters such as magnetic $(M)$, power-law index $(m)$, stretching ratio $(\epsilon)$, and surface thickness $(s)$ in the following sub-section. The numerical calculations have been carried out for a wide range of values of the mentioned parameters; $\mathrm{M}(1.0 \leq \mathrm{M} \leq 10.0), \mathrm{m}(0.0 \leq \mathrm{m} \leq 3.0), \varepsilon(0.0 \leq \varepsilon \leq 2.0), \quad s(0.05 \leq s \leq 2.0)$, $\operatorname{Pr}(0.71 \leq \operatorname{Pr} \leq 7.0)$.

\section{Velocity Profiles and Skin Friction Coefficient}

Figure $2 \mathrm{a}$ and Figure $2 \mathrm{~b}$ represent the influence of magnetic parameter on viscous fluid velocity profile for thinner $(s=0.05)$ and thicker surfaces $(s=2.0)$ when $\varepsilon<1, \epsilon=1.0$ and $\varepsilon>1$ respectively. It is noticed from these figures that the variation of the stretching ratio parameter has a significant effect on the velocity profile in connection with the magnetic parameter. These plots depict that the velocity profile of the viscous fluid and boundary layer thickness affected significantly with increasing magnetic parameters. From these figures, it is seen that the velocity profile of the viscous fluid and boundary layer thickness squeezes when $\varepsilon>1$ and the fluid flow form an inverted boundary layer pattern but in the case of $\varepsilon<1$, the reverse trend occurs in velocity profile both of the thinner and thicker surfaces of the bullet-shaped object. This happens because in presence of 
the term $\frac{\sigma B_{0}^{2}}{\rho}\left(U_{\infty}-u\right)$ in equation (2) which is the combination of pressure force $\left(\frac{\sigma B_{0}^{2}}{\rho} U_{\infty}\right)$ and Lorentz force $\left(\frac{\sigma B_{0}^{2}}{\rho} u\right)$ terms.

Figure 2. Effect of Magnetic Parameter $(M)$ on Velocity Profile Taking (a) $s=$ 0.05 and $\epsilon=0.0$ (Lower Panel), $\epsilon=1.0$ (Separation Line), $\epsilon=2.0$ (Upper Panel), and (b) $s=2.0$ and $\epsilon=0.0$ (Lower Panel), $\epsilon=1.0$ (Separation Line), $\epsilon=2.0$ (Upper Panel)

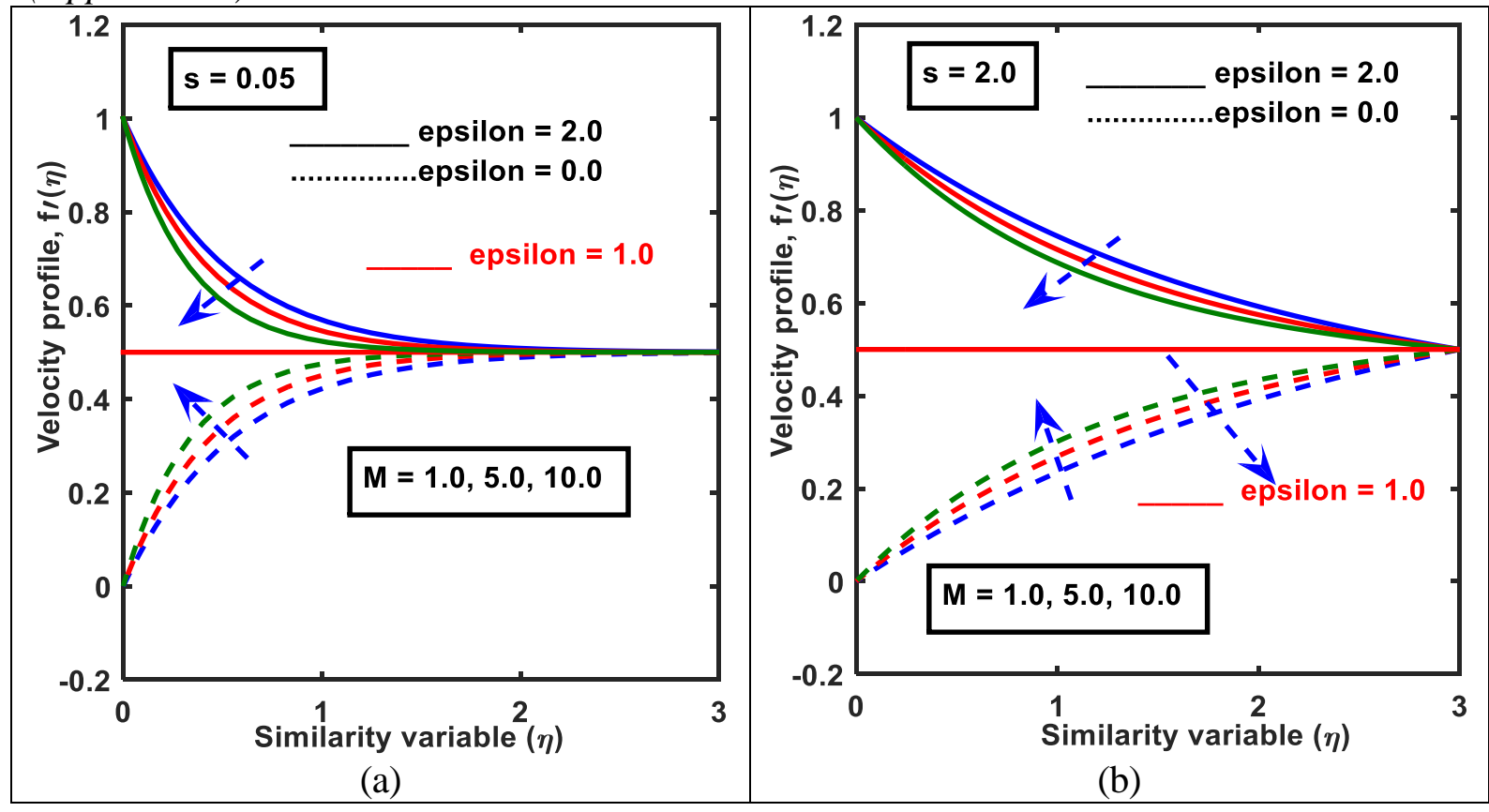

The velocity profile squeezes when Lorentz's force is greater than the pressure force and vice-versa. Again, it is also seen that the surface velocity and the free stream velocity are equal in the case of $\epsilon=1.0$. It is also observed that, if the surface thickness parameter $(s)$ reduces then the fluid flow form a certain boundary layer structure and the fluid velocity satisfies the far-field boundary condition. On the other hand, while surface thickness parameter $(s)$ increases then the fluid flow does not satisfy the far-field boundary condition and the boundary layer structure has no definite shape. Therefore, the velocity boundary layer thickness is higher in the case of a thicker bullet-shaped object $(s=2.0)$ than the thinner bullet-shaped object $(s=0.05)$.

Figures $3 \mathrm{a}$ and $3 \mathrm{~b}$ display the skin friction coefficient for the effect of the magnetic parameter $(M)$ with stretching ratio parameter $\varepsilon<1$ and $\varepsilon>1$, and the surface thickness parameter $s=0.05, s=1.0$, respectively. From these figures, it is observed that the skin friction coefficient enhances in the case of $\varepsilon<1$ but reduces while $\varepsilon>1$ for both of the thinner and thicker surfaces of the bullet-shaped object. It is observed that the skin friction coefficient is inversely proportional to the dimensionless velocity gradient. The velocity gradient at the surface is positive 
when $\varepsilon<1$ and negative due to $\varepsilon>1$ but zero while $\epsilon=1.0$ for all values of the controlling parameters. The positive values of the velocity gradient indicate that the fluid applies a drag force on the bullet-shaped object, on the other hand, the bullet-shaped object exerts a drag force on the fluid flow while the velocity gradient is negative.

Figure 3. Effect of Magnetic Parameter (M) on Skin Friction Coefficient Taking (a) $s=0.05$ and $\epsilon=0.0$ (Upper Panel), $\epsilon=2.0$ (Lower Panel), and (b) $s=2.0$ and $\epsilon=0.0$ (Upper Panel), $\epsilon=2.0$ (Lower Panel)

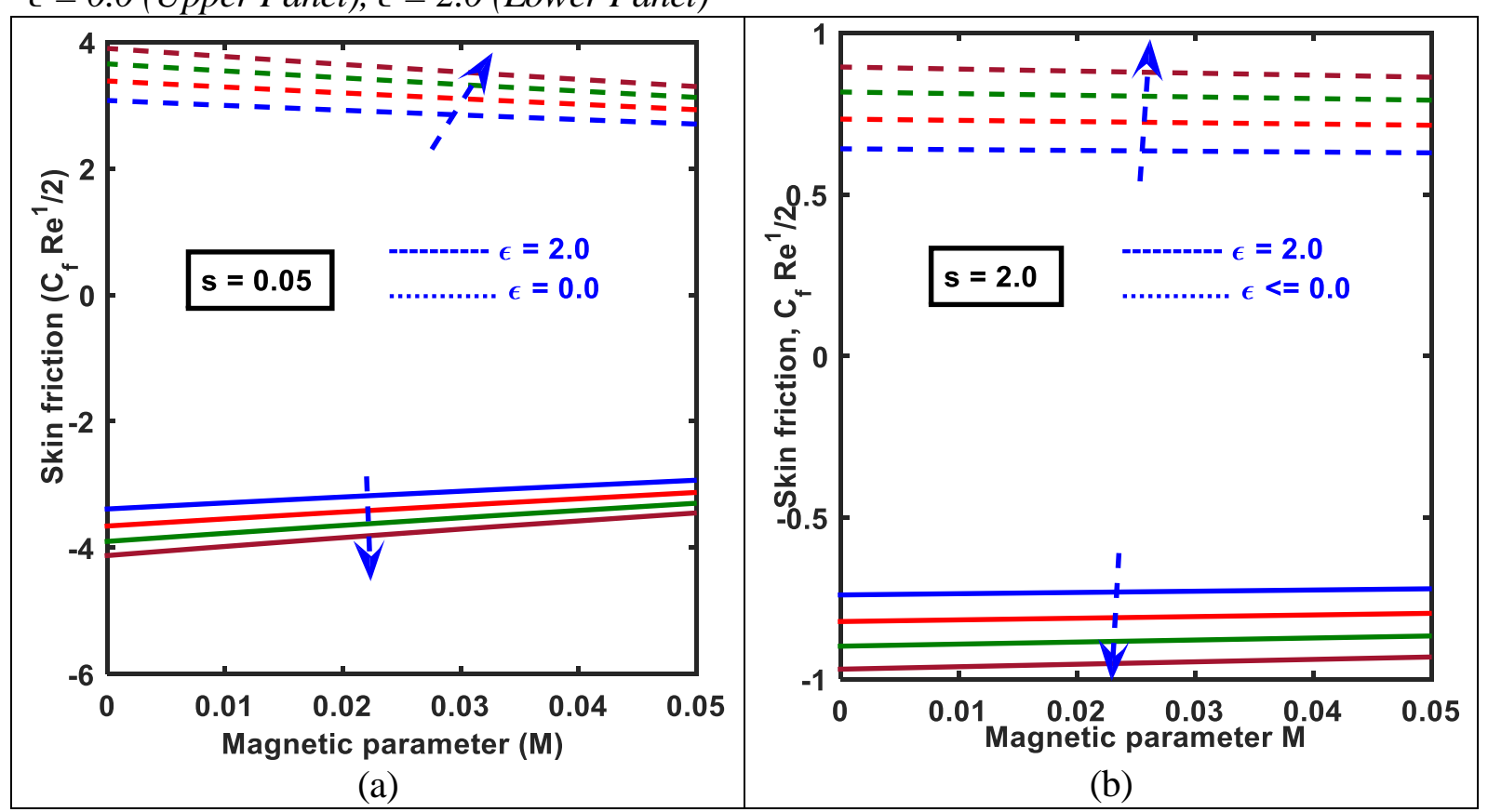

Therefore, the parameter $M$ is directly proportional to skin friction in the case of $\varepsilon<1$ but inversely proportional when $\varepsilon>1$. It is observed from Table 1 that, when $M$ changes from 1.0 to 10.0 for $s=0.05$ and $\epsilon=0.0$, the skin friction decreases $25.5 \%$ whereas for $s=0.05$ and $\epsilon=2.0$ the skin friction increases $22.13 \%$. Hence, the skin friction is higher for the non-stretching surface $(\varepsilon=0)$ than the stretching surface $(\varepsilon>0)$.

Figures $4 \mathrm{a}$ and $4 \mathrm{~b}$ show the variation of a velocity profile for the effect of power-law index parameter when the stretching ratio parameter $\varepsilon<1$ and $\varepsilon>1$, and the surface thickness parameter $s=0.05, s=2.0$, respectively. 
Figure 4. Influence of Power-Law Index Parameter $(m)$ on Velocity Profile Taking (a) $s=0.05$ and $\epsilon=0.0$ (Lower Panel), $\epsilon=1.0$ (Separation Line), $\epsilon=2.0$ (Upper Panel), and (b) $s=2.0$ and $\epsilon=0.0$ (Lower Panel), $\epsilon=1.0$ (Separation Line), $\epsilon=$ 2.0 (Upper Panel)

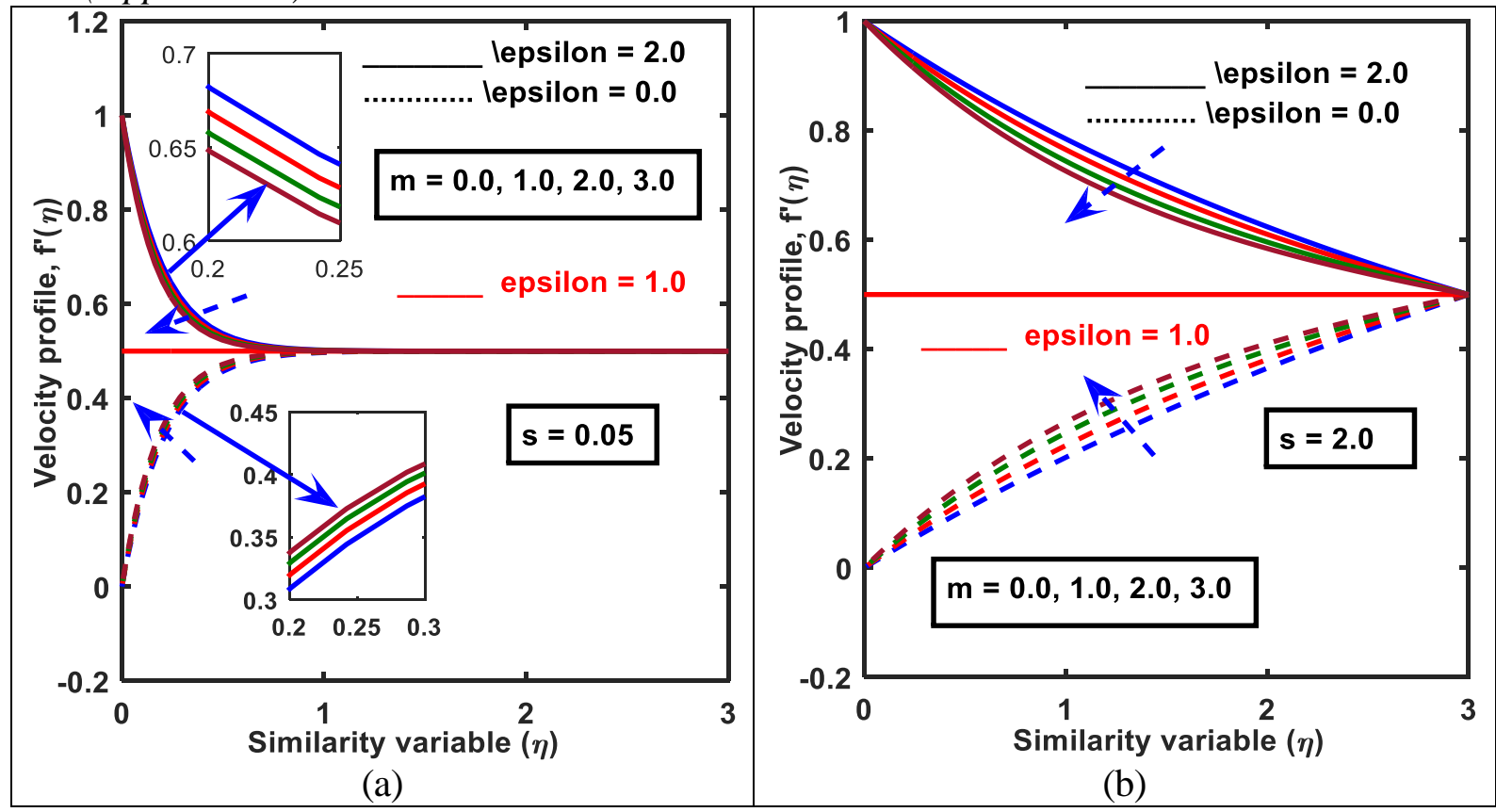

These plots represent that the velocity profile of the viscous fluid and boundary layer thickness affected insignificantly with an increasing power-law index parameter due to thinner surface $(s=0.05)$ whereas the reverse trend happens in the case of thicker surface $(s=2.0)$. From these figures, it is noticed that the velocity profile and boundary layer thickness squeezes when $\varepsilon>1$ and the fluid flow form an inverted boundary layer pattern whereas, in the case of $\varepsilon<1$, the velocity profile expands but boundary layer thickness squeezes the and fluid flow form a certain boundary layer pattern. It is also observed that the velocity profile and boundary layer thickness satisfy the far-field boundary condition in the case of a thinner surface $(s=0.05)$. On the other hand, due to the thicker surface $(s=2.0)$, the velocity profile, and boundary layer thickness are not in parabolic shapes, so this is insignificant with the physical behavior of the boundary layer concept because in this case, the boundary condition does not satisfy. Therefore, the velocity boundary layer thickness is higher in the case of a thicker bullet-shaped object $(s=2.0)$ than a thinner bullet-shaped object $(s=0.05)$.

Figure $5 \mathrm{a}$ and $5 \mathrm{~b}$ display the skin friction coefficient for the effect of powerlaw index parameter $(m)$ by taking the stretching ratio parameter $\varepsilon<1$ and $\varepsilon>1$, and the surface thickness parameter $s=0.05, s=1.0$, respectively. From these figures, it is seen that the skin friction coefficient enhances in the case of $\varepsilon<1$ but reduces while $\varepsilon>1$ for both of the thinner and thicker surfaces. 
Figure 5. Effect of Power-Law Index Parameter $(m)$ on Skin Friction Coefficient Taking (a) $s=0.05$ and $\epsilon=0.0$ (Lower Panel), $\epsilon=2.0$ (Upper Panel), and (b) $s=$ 2.0 and $\epsilon=0.0$ (Lower Panel), $\epsilon=2.0$ (Upper Panel)

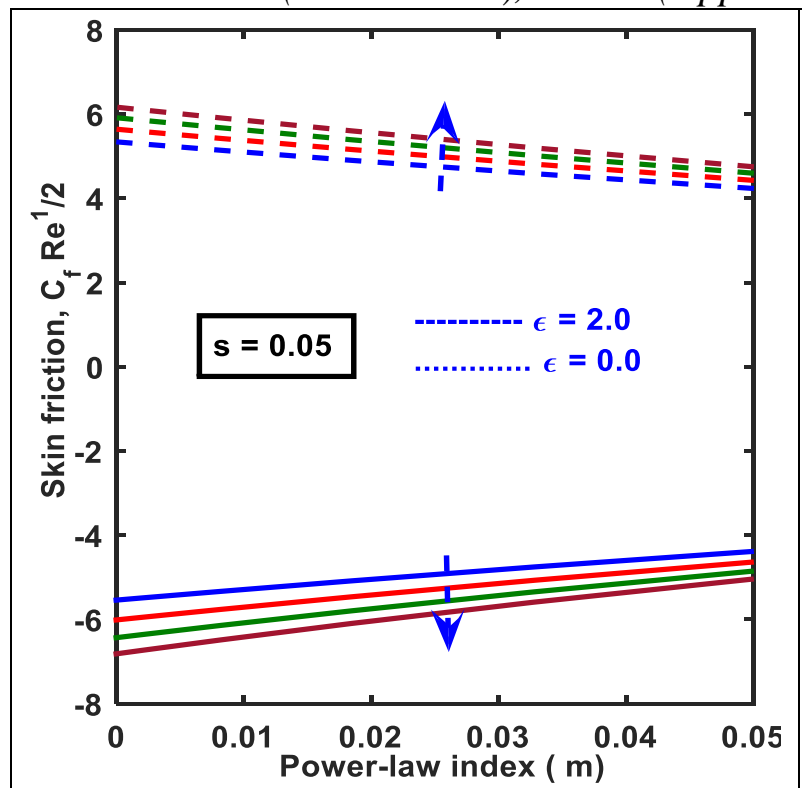

(a)

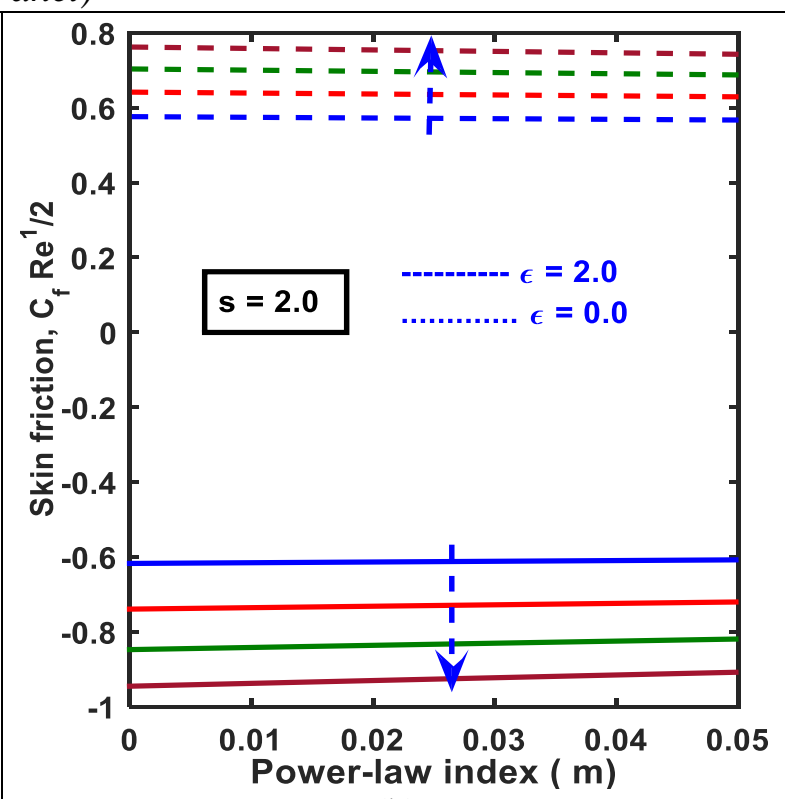

(b)

These graphs also show that the velocity gradient at the surface is a positive when $\varepsilon<1$ and negative while $\varepsilon>1$ but zero while $\epsilon=1.0$ for all values of the controlling parameters. Therefore, the positive values $f^{\prime \prime}(\eta)$ indicate that the fluid applies a drag force on the bullet-shaped object and the negative value indicates the bullet-shaped object exerts a drag force on the fluid flow. It is observed from Table 1 that, when $m$ changes from 0.0 to 2.0 for $s=0.05$ and $\epsilon=0.0$, the skin friction decreases $10.73 \%$ whereas for $s=0.05$ and $\epsilon=2.0$ the skin friction increases $16.1 \%$.

Figures $6 \mathrm{a}$ and $6 \mathrm{~b}$ illustrate the effects of surface thickness parameter $(s)$ $(0.05 \leq s \leq 2.0)$ on the velocity distribution and skin friction coefficient when the other parameters are fixed by considering the stretching ratio parameter $\varepsilon<1$ and $\varepsilon>1$, and the surface thickness parameter $s=0.05, s=2.0$, respectively. These plots show that the velocity profile of the viscous fluid and boundary layer thickness affected significantly with increasing surface thickness parameters. From Figure 6(a) it is seen that the velocity profile and boundary layer thickness expands when $\varepsilon>1$ and the fluid flow form an inverted boundary layer pattern but in the case of $\varepsilon<1$ the velocity profile squeezes but velocity boundary layer thickness increases and the fluid flow forms a definite boundary layer pattern. This happens because the increase in shape and size of the bullet-shaped object enhances the surface area of the object that provides more resistance against the fluid motion as a result the fluid velocity decreases. Therefore, the velocity boundary layer thickness is higher in the case of a thicker bullet-shaped object $(s=2.0)$ than the thinner bullet-shaped object ( $s=0.05$ ). From Figure $6(\mathrm{~b})$, it is noticed that the skin friction coefficient reduces in the case of $\varepsilon<1$ but increases while $\varepsilon>1$ for both of 
the thinner and thicker surfaces. Hence, the parameter $s$ is inversely proportional to $C_{f} \sqrt{\mathrm{Re}_{x}}$ in the case of $\varepsilon<1$ but directly proportional when $\varepsilon>1$. This graph also shows that the velocity gradient at the surface is a positive when $\varepsilon<1$ and negative while $\varepsilon>1$ but zero while $\epsilon=1.0$ for all values of the controlling parameters. Therefore, the fluid applies a drag force on the bullet-shaped object for the positive values $f^{\prime \prime}(\eta)$ while the bullet-shaped object exerts a drag force on the fluid flow for the negative values $f^{\prime \prime}(\eta)$. It is observed from Table 1 that, when $s$ changes from 0.05 to 0.3 for $\epsilon=0.0$, the skin friction increases $76.5 \%$ whereas for $\epsilon=2.0$ the skin friction decreases $75.2 \%$. Hence, the skin friction is higher for $\epsilon=$ 0.0 than $\epsilon=2.0$.

Figure 6. Effect of Surface Thickness Parameter (s) on (a) Velocity Profile for $\epsilon=$ 0.0 (Lower Panel), 1.0 (Separation Line), 2.0 (Upper Panel), and (b) Skin Friction for $\epsilon=0.0$ (Lower Panel), 2.0 (Upper Panel)

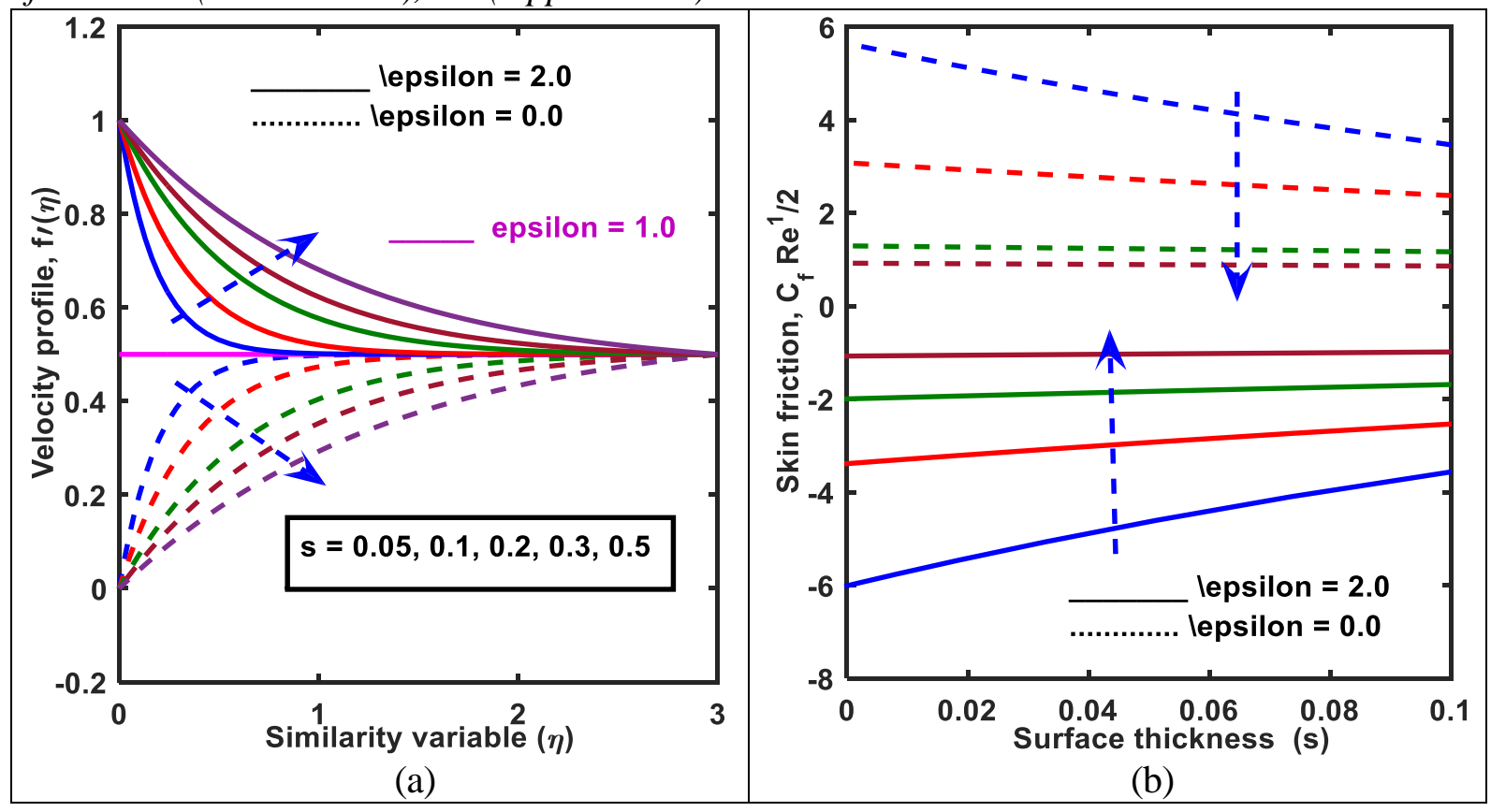

Figure $7 \mathrm{a}$ and $7 \mathrm{~b}$ illustrate the velocity profile for the increasing effect of stretching ratio parameter $(0 \leq \varepsilon \leq 2.0)$ consider the surface thickness parameter $s=0.05$ and $s=2.0$, respectively. From these figures, it is noticed that the velocity profile and boundary layer thickness expands when $\varepsilon>1$ and the fluid flow form an inverted boundary layer pattern but in the case of $\varepsilon<1$ the fluid flow form a certain boundary layer pattern. It is also observed that the velocity profile and boundary layer thickness satisfy the far-field boundary condition in the case of a thinner surface $(s=0.05)$. On the other hand, while the thicker surface $(s=2.0)$, the velocity profile is not in a parabolic shape, this is insignificant with the physical behavior because in this case, the boundary condition does not satisfy by the velocity profile. Therefore, the velocity boundary layer thickness is higher in 
the case of the thicker bullet-shaped object $(s=2.0)$ than the thinner bullet-shaped object $(s=0.05)$.

Figure 7. Effect of Stretching Ratio Parameter $(\epsilon)$ on Velocity Profile Taking $(a) s$ $=0.05$ and $\epsilon=0.0$ (Lower Panel), $\epsilon=1.0$ (Separation Line), $\epsilon=2.0$ (Upper Panel), and $(b) s=2.0$ and $\epsilon=0.0$ (Lower Panel), $\epsilon=1.0$ (Separation Line), $\epsilon=2.0$ (Upper Panel)

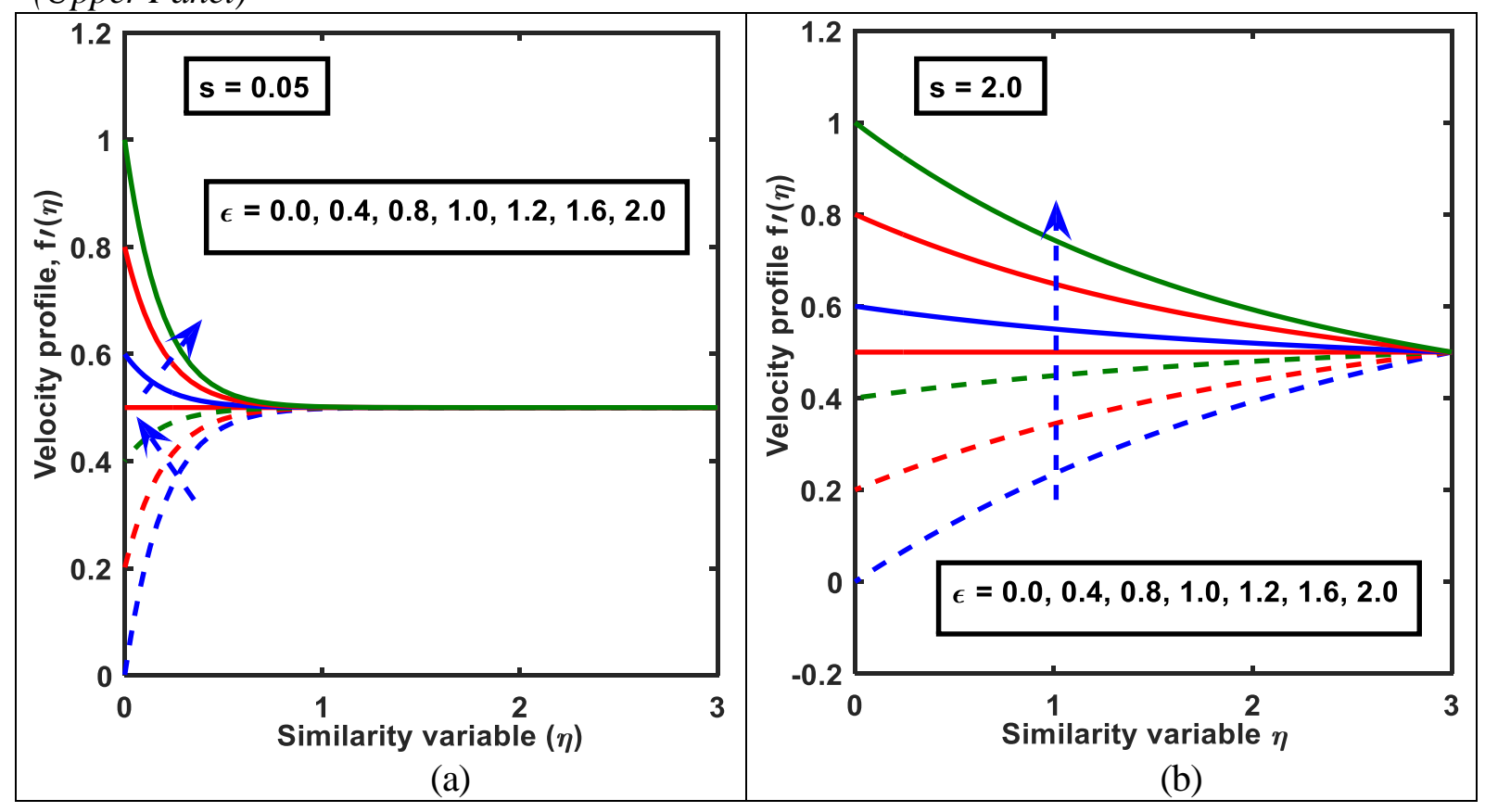

From these figures, it is observed that the velocity profile and momentum boundary layer thickness is getting expand due to power-law index parameter $(m)$, surface thickness parameter $(s)$, and stretching ratio parameter $(\epsilon)$ whereas magnetic parameter $(M)$ has a reverse effect on it in the case of $\varepsilon>1$ for both of the thicker $(s=2.0)$ and thinner $(s=0.05)$ surfaces. On the other hand, in the case of $\varepsilon<1$ the velocity profile and momentum boundary layer thickness are getting expand due to magnetic parameter $(M)$, power-law index parameter $(m)$, and stretching ratio parameter $(\epsilon)$ but squeezes for the surface thickness parameter $(s)$. Again, it is also revealed that in the case of a thicker bullet-shaped object $(s=2.0)$ the velocity profile does not approach the ambient condition asymptotically but intersects the vertical axis with a steep angle and the boundary layer structure has no definite shape whereas in the case of a thinner bullet-shaped object $(s=0.05)$ the velocity profile converge the ambient condition asymptotically and the boundary layer structure has a definite shape. It is also noticed that the thinner bullet-shaped object represents a thinner momentum boundary layer than the thicker bullet-shaped object in both static and moving bullet-shaped objects.

\section{Temperature Profiles and Nusselt Number}

Figures $8 \mathrm{a}$ and $8 \mathrm{~b}$ depicts the variation of Prandtl number $(\operatorname{Pr})(0.71 \leq \operatorname{Pr} \leq 10.0)$ on temperature profile when stretching ratio parameter $\varepsilon<1$ or $>1$ and the surface 
thickness parameter $s=0.05, s=2.0$, respectively. These plots represent that the temperature profile of the viscous fluid and thermal boundary layer thickness affected significantly with increasing Prandtl number.

Figure 8. Temperature Profile for Different Values of Prandtl Number when (a) $\epsilon$ $=0, s=0.05, s=2.0$, and $(b) \epsilon=2.0, s=0.05, s=2.0$

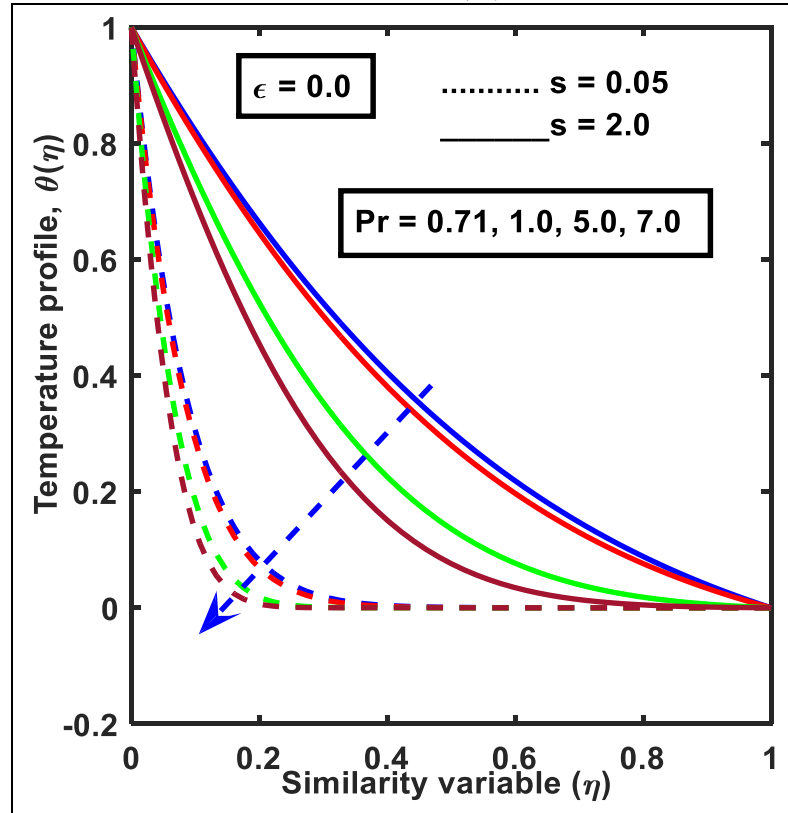

(a)

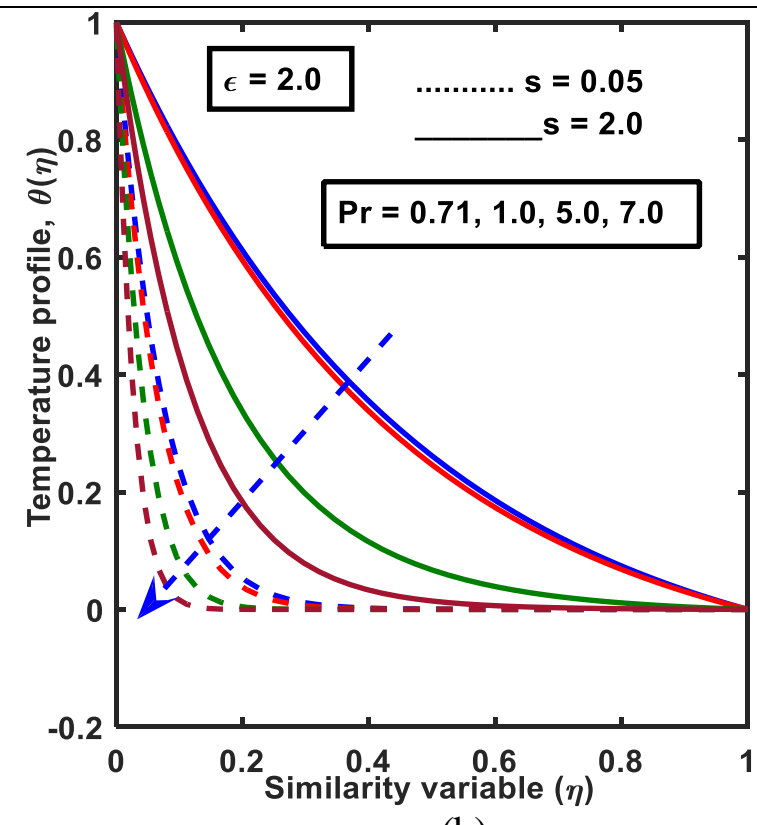

(b)

It is seen from these figures that the variation of surface thickness parameter has a significant effect on fluid temperature in connection with the Prandtl number. From these figures, it is seen that the temperature profile and boundary layer thickness squeezes for both the thicker $(s=2.0$, and thinner $(s=0.05)$ surfaces of the bullet-shaped object. It is also observed that the thermal boundary layer thickness is higher in the case of the thicker surface $(s=2.0)$ than the thinner surface $(s=0.05)$. This happens because a smaller Prandtl number means heat is quickly diffuse from the heated surface than for higher values of Pr. For increasing values of Prandtl number, the temperature profiles are closing to the solid surface. So, for cooling purposes, the Prandtl number can be used. A smaller surface thickness has a greater decelerating effect than a thicker surface. Therefore, it is noticed that for higher values of surface thickness parameter, the maximum temperature shows in the thermal boundary layer compared to that of lower surface thickness parameter.

Figure $9 \mathrm{a}$ and Figure $9 \mathrm{~b}$ depict the influence of power-law index parameter on viscous fluid temperature for thinner surface $(\mathrm{s}=0.05)$ and thicker surface $(\mathrm{s}=$ 2.0) when $\epsilon=0.2$ and 2.0 respectively. It is noticed from these figures that the variation of the surface thickness parameter has a significant effect on viscous fluid temperature in connection with the power-law index parameter. A lower surface thickness parameter has a greater decelerating effect. For thicker surfaces ( $\mathrm{s}=2.0$ ) with increasing $\mathrm{m}$, the decelerating effect is more prominent than for thinner surfaces $(\mathrm{s}=0.05)$. Therefore, the thermal boundary layer thickness is 
higher due to the thicker surface when compared with the thinner surface. Figure 9c and Figure 9d also represent the influence of power-law index parameter on viscous fluid temperature for air $(\operatorname{Pr}=0.71)$ and water $(\operatorname{Pr}=7.0)$ when $\mathrm{s}=0.05$ and 2.0 respectively.

Figure 9. Temperature Profile for Different Values of Power-Law Index Parameter (m) when (a) $\epsilon=0.2, s=0.05, s=2.0,(b) \epsilon=2.0, s=0.05, s=2.0$ (c) $s=0.2, P r$ $=0.71, \operatorname{Pr}=7.0$, and $(d) s=2.0, \operatorname{Pr}=0.71, \operatorname{Pr}=7.0$

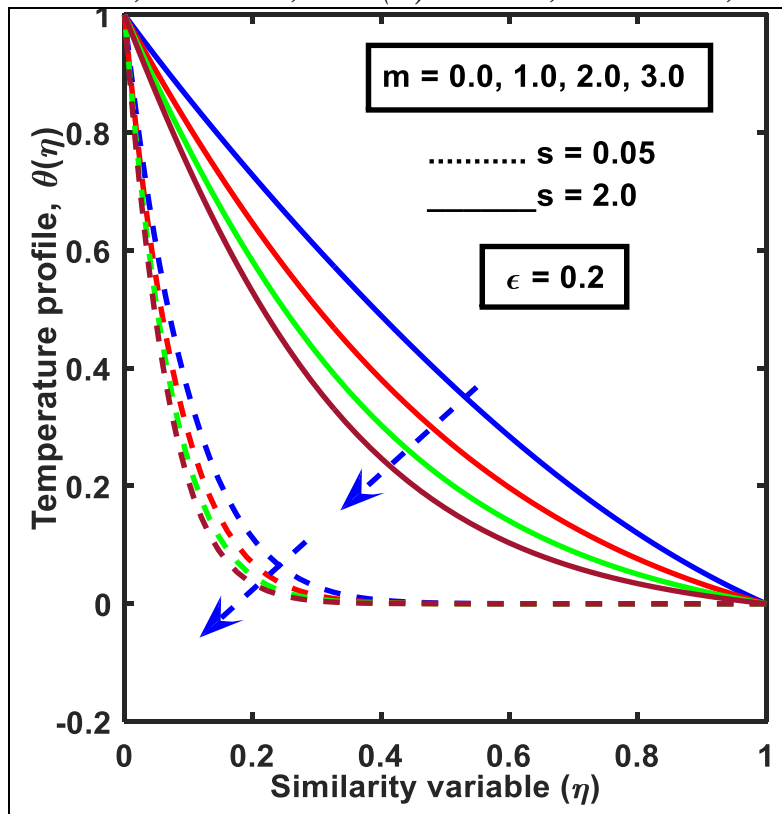

(a)

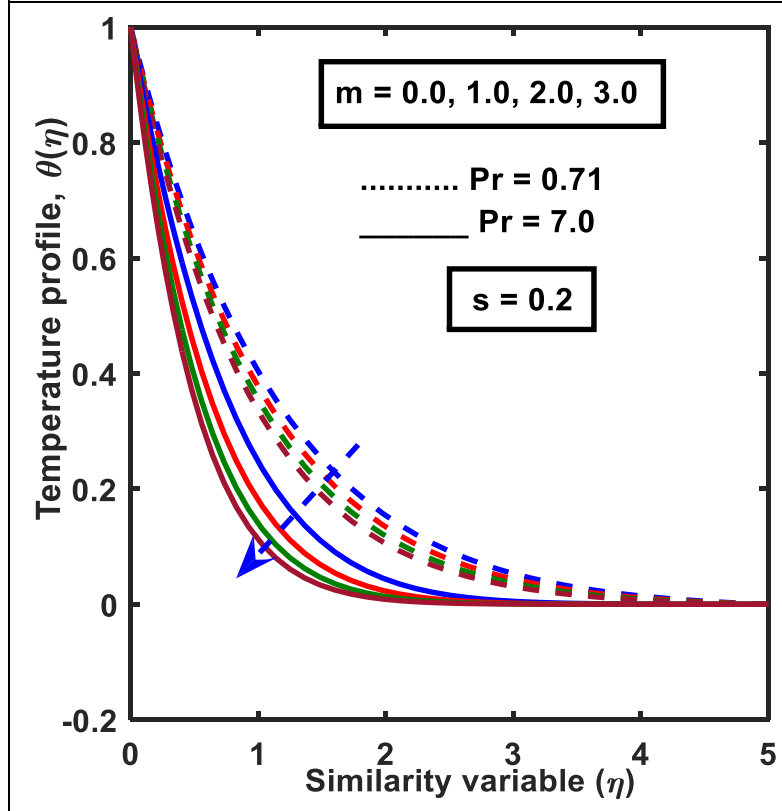

(c)

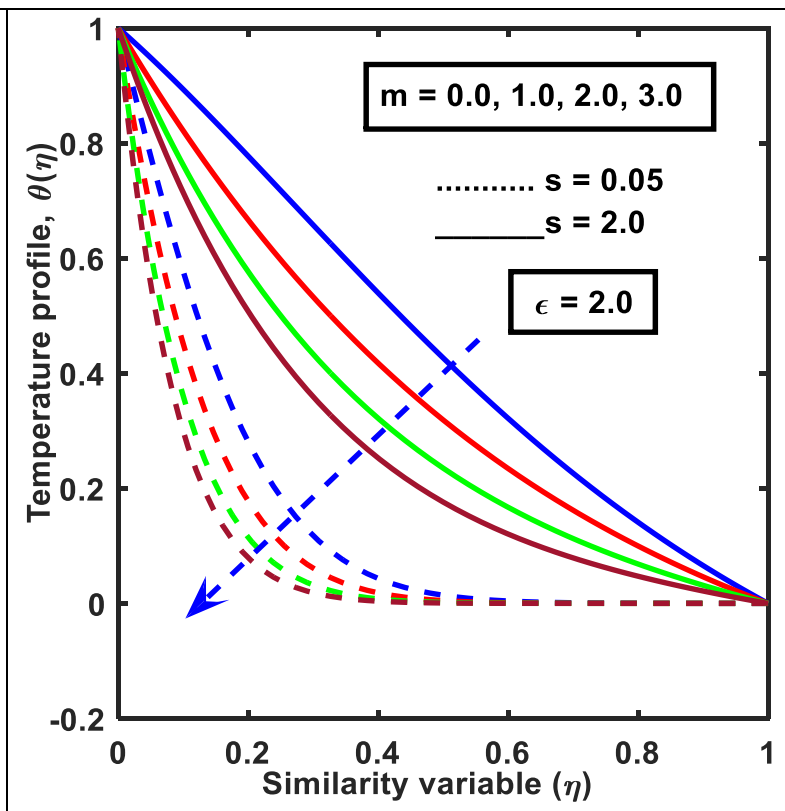

(b)

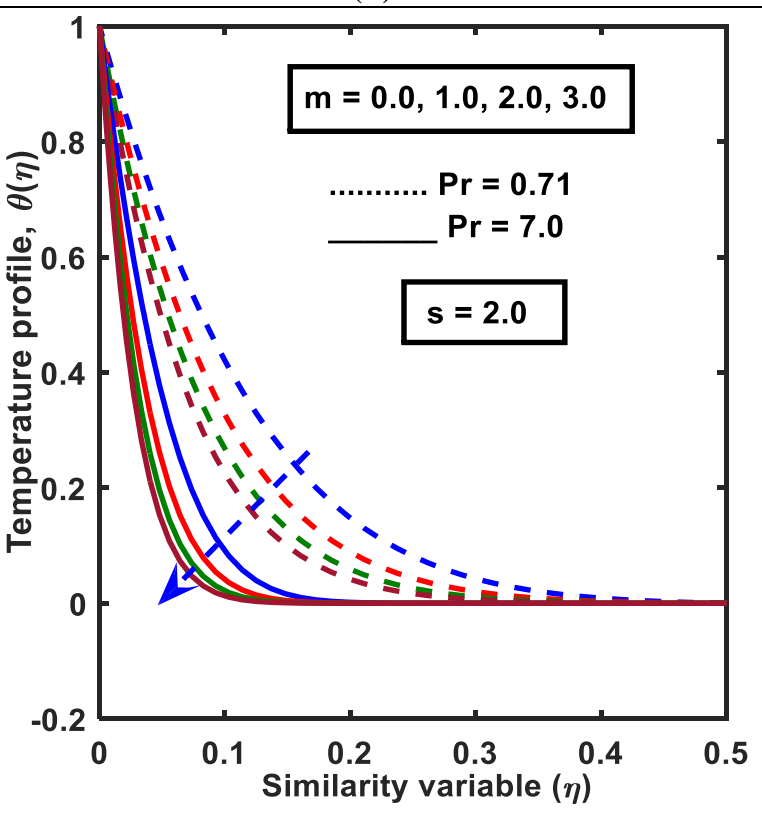

(d) 
It is seen from these figures that the variation of the Prandtl number has a significant effect on viscous fluid temperature in connection with the power-law index parameter.

A higher Prandtl number has a greater decelerating effect. For air $(\operatorname{Pr}=0.71)$ with increasing $\mathrm{m}$, the decelerating effect is more prominent than for water $(\mathrm{Pr}=$ 7.0). Therefore, the thermal boundary layer thickness is higher for air when compared with water. From these figures, the temperature distribution and also the thermal boundary layer thickness decrease with the increase of $m$ for all mentioned cases. These plots also represent that the temperature profile of the viscous fluid and thermal boundary layer thickness affected significantly with increasing powerlaw index parameter. Therefore, it is seen that for higher values of surface thickness parameter, the maximum temperature shows in the thermal boundary layer compared to that of lower surface thickness parameter. Again, in the case of air $(\operatorname{Pr}=0.71)$, the highest temperature shows in the thermal boundary layer compared to that of water $(\operatorname{Pr}=7.0)$.

Figure 10a and Figure 10b portray the influence of surface thickness parameter $(s)$ on viscous fluid temperature for air $(\operatorname{Pr}=0.71)$ and water $(\operatorname{Pr}=7.0)$ when $\epsilon=0.2$ and 2.0 respectively. It shows that the temperature profile and thermal boundary layer thickness expand with an increase of surface thickness parameter because the heat transfer rate enhances.

Figure 10. Temperature Profile for Different Values of Surface Thickness Parameter $(s)$ when (a) $\epsilon=0.2, \operatorname{Pr}=0.71,7.0$ and $(b) \epsilon=2.0, \operatorname{Pr}=0.71,7.0$

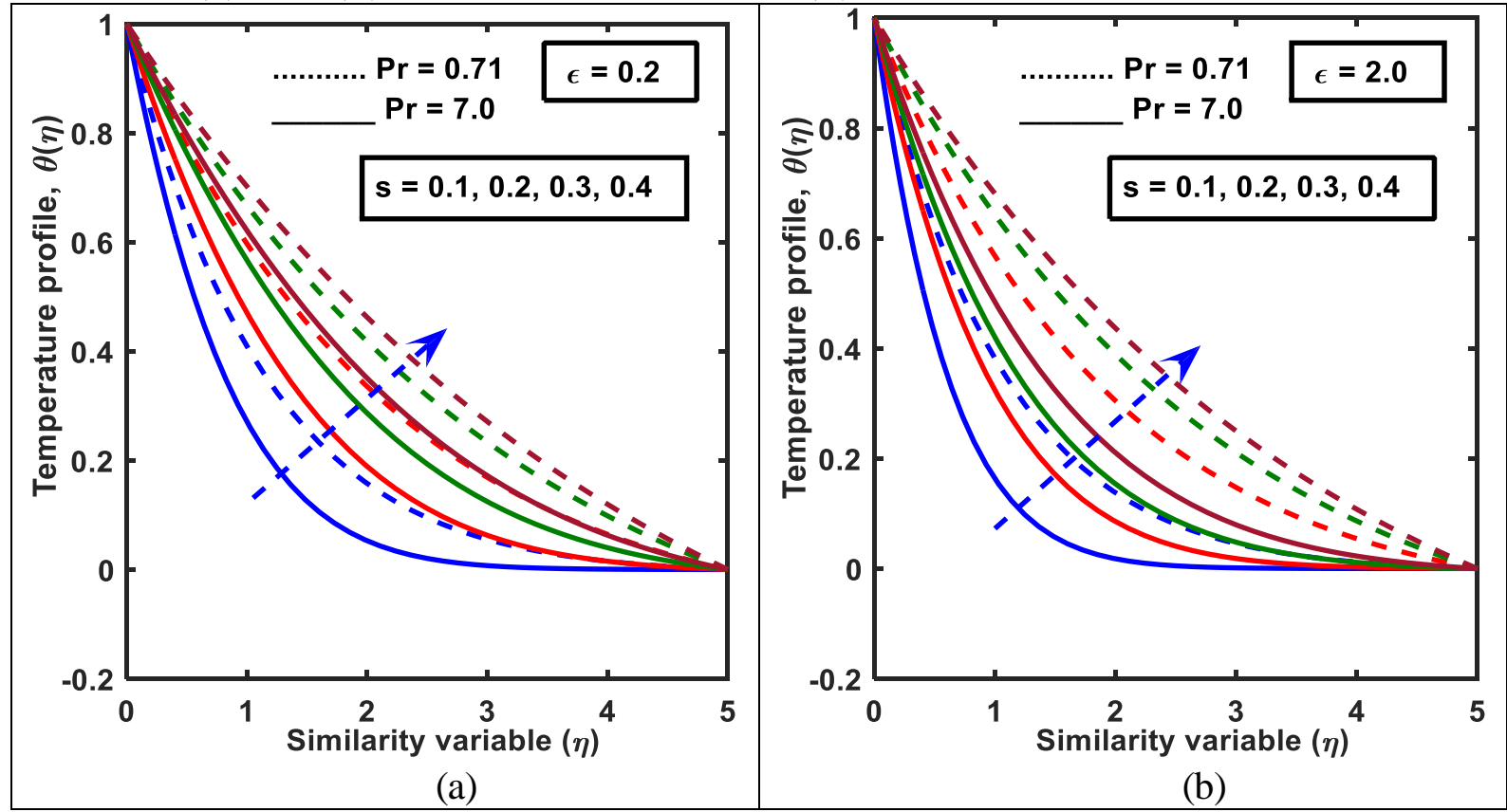

It is seen from these figures that the variation of the Prandtl number has a significant effect on viscous fluid temperature in connection with the surface thickness parameter. A higher Prandtl number has a greater decelerating effect than a lower Prandtl number. For air $(\operatorname{Pr}=0.71)$ with increasing $s$, the accelerating effect is more prominent than for water $(\operatorname{Pr}=7.0)$. Therefore, the thermal 
boundary layer thickness is higher for air when compared with water. Again, in the case of air $(\operatorname{Pr}=0.71)$, the highest temperature shows in the thermal boundary layer compared to that of water $(\operatorname{Pr}=7.0)$.

Figure 11a and Figure 11b display the effects of the stretching ratio parameter $(\epsilon)$ on viscous fluid temperature for air $(\operatorname{Pr}=0.71)$ and water $(\operatorname{Pr}=7.0)$ when $s=$ 0.05 and 2.0 respectively. It shows that the temperature profile and thermal boundary layer thickness squeeze with an increase of the stretching ratio parameter because the velocity profile increases for the effect of this parameter which tends to pass more fluid from the boundary layer region to the surrounding fluid.

Figure 11. Temperature Profile for Different Values of Stretching Ratio Parameter ( $\epsilon$ ) when (a) $s=0.2, \operatorname{Pr}=0.71, \operatorname{Pr}=7.0$ and $(b) s=2.0, \operatorname{Pr}=0.71, \operatorname{Pr}=7.0$

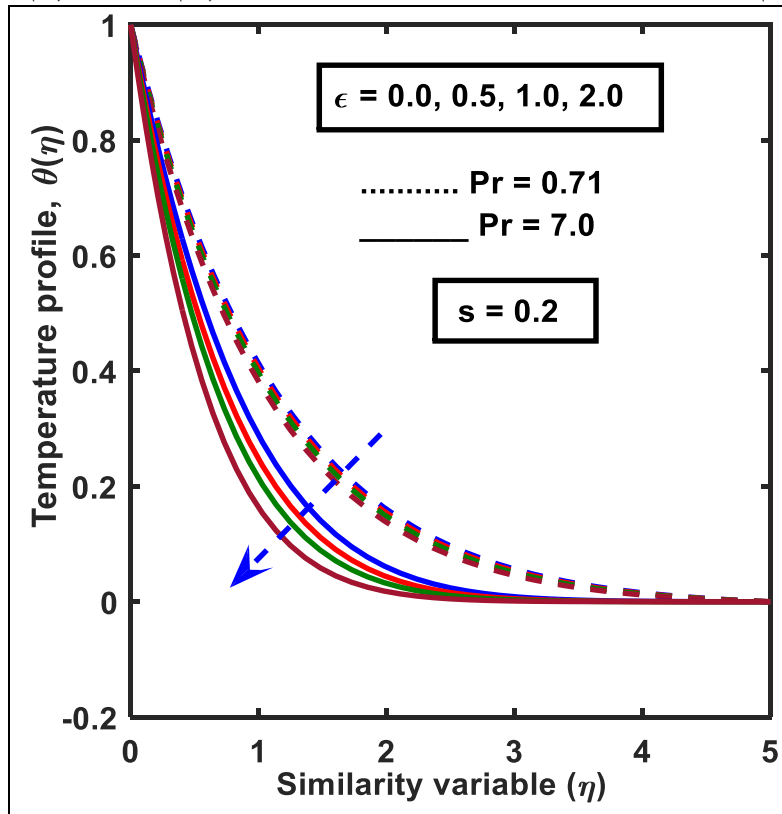

(a)

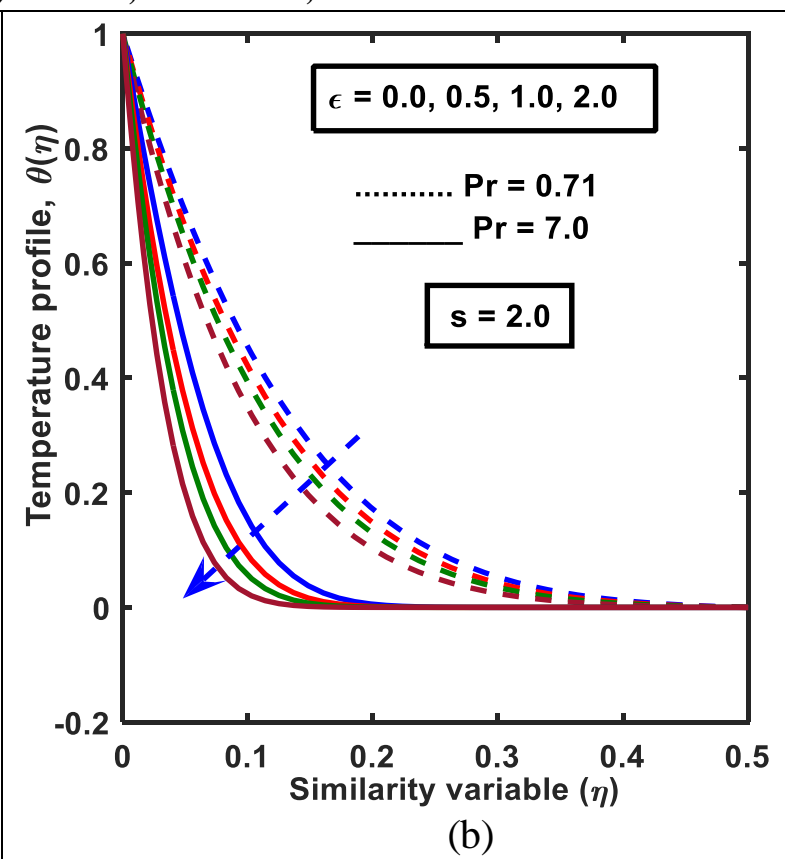

(b)

It is seen from these figures that the variation of the Prandtl number has a significant effect on viscous fluid temperature in connection with the stretching ratio parameter. A higher Prandtl number has a greater decelerating effect. For water $(\operatorname{Pr}=7.0)$ with increasing the stretching ratio parameter, the decelerating effect is more prominent than for air $(\operatorname{Pr}=0.71)$. Again, in the case of air ( $\operatorname{Pr}=$ 0.71), the highest temperature shows in the thermal boundary layer compared to that of water $(\operatorname{Pr}=7.0)$.

\section{Effect of Heat Generation Parameter $\left(Q^{*}\right)$ on Temperature Profile and Nusselt} Number

Figures 12a-12f display the effect of heat generation parameter on the temperature profile and Nusselt number when the stretching ratio parameter $\epsilon=$ $0.2, \epsilon=2.0$, the surface thickness parameter $s=0.05, s=2.0$, and $\operatorname{Pr}=0.71,7.0$ respectively. Figure 12a and Figure 12b depict the influence of heat generation parameter on viscous fluid temperature for thinner surface $(s=0.05)$ and thicker 
surface $(\mathrm{s}=2.0)$ when $\epsilon=0.2$ and 2.0 respectively. These plots represent that the temperature profile of the viscous fluid and thermal boundary layer thickness affected significantly with increasing heat generation parameters. It is noticed from these figures that the variation of heat generation parameter has a significant effect on fluid temperature in connection with the surface thickness parameter and the Prandtl number. A lower surface thickness parameter has a lower accelerating effect. For thicker surfaces $(\mathrm{s}=2.0)$ with increasing $\mathrm{Q}^{*}$, the accelerating effect is more prominent than for thinner surfaces $(s=0.05)$. Therefore, the thermal boundary layer thickness is higher due to the thicker surface when compared with the thinner surface. Therefore, the temperature profile and boundary layer thickness expand for both of the thicker $(s=2.0)$ and thinner $(s=0.05)$ surfaces of the bullet-shaped object whereas the Nusselt number decreases (Figures 12e and 12f). Figures $12 \mathrm{c}$ and $12 \mathrm{~d}$ also represent the influence of heat generation parameter on viscous fluid temperature for air $(\operatorname{Pr}=0.71)$ and water $(\operatorname{Pr}=7.0)$ when $\mathrm{s}=0.05$ and 2.0 respectively. It is seen from these figures that the variation of the Prandtl number has a significant effect on viscous fluid temperature in connection with the heat generation parameter. A higher Prandtl number has a greater accelerating effect on the temperature profile. For water $(\operatorname{Pr}=0.71)$ with increasing $\mathrm{Q}^{*}$, the accelerating effect is more prominent than for air $(\operatorname{Pr}=0.71)$. Therefore, the thermal boundary layer thickness is higher for water when compared with air. However, the temperature attains its minimum for a low value of Prandtl number $(\operatorname{Pr}=0.71)$ and a higher value of Prandtl number $(\operatorname{Pr}=7.0)$ in connection with heat generation parameter shooting up the profiles near the surface of the bullet-shaped object within the region $\eta<0.4$ and then the profile decreases sharply to meet the boundary condition as well. From these figures, the temperature profile and thermal boundary layer thickness increase with the increase of $Q^{*}$ for all mentioned cases. Therefore, it is seen that for higher values of surface thickness parameter, the maximum temperature shows in the thermal boundary layer compared to that of lower surface thickness parameter. Again, in the case of water $(\operatorname{Pr}=7.0)$, the highest temperature shows in the thermal boundary layer compared to that of air $(\mathrm{Pr}=0.71)$. It happens because heat sources produce more heat energy near the surface as a result the temperature of the fluid increases. 
Figure 12. Effect of Heat Generation $Q^{*}$ on Temperature Profile and Local Nusselt Number when (a) $\epsilon=0.2, s=0.05, s=2.0(b) \epsilon=2.0, s=0.05, s=2.0$ (c) $s=0.05, \operatorname{Pr}=0.71, \operatorname{Pr}=7.0(d) s=2.0, \operatorname{Pr}=0.71, \operatorname{Pr}=7.0(e) \epsilon=0.2, s=$ $0.05, \operatorname{Pr}=0.71$, and $(f) \epsilon=2.0, s=0.05, \operatorname{Pr}=0.71$

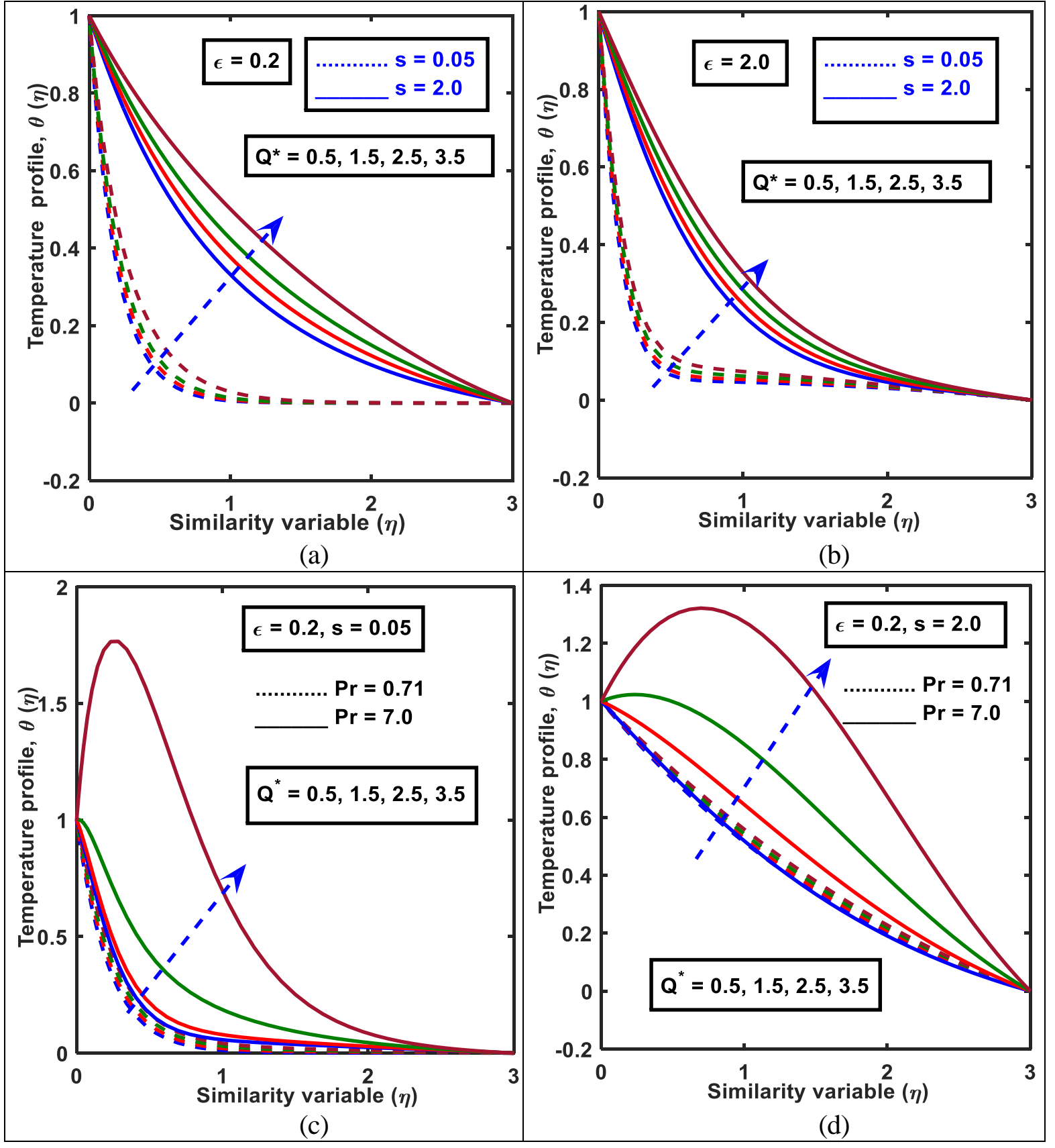




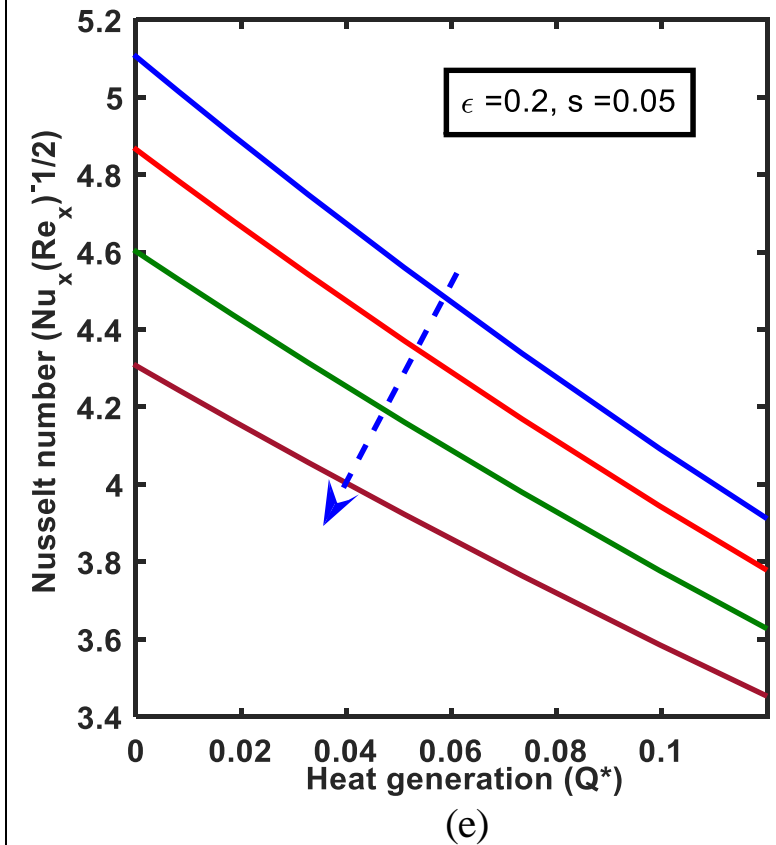

(e)

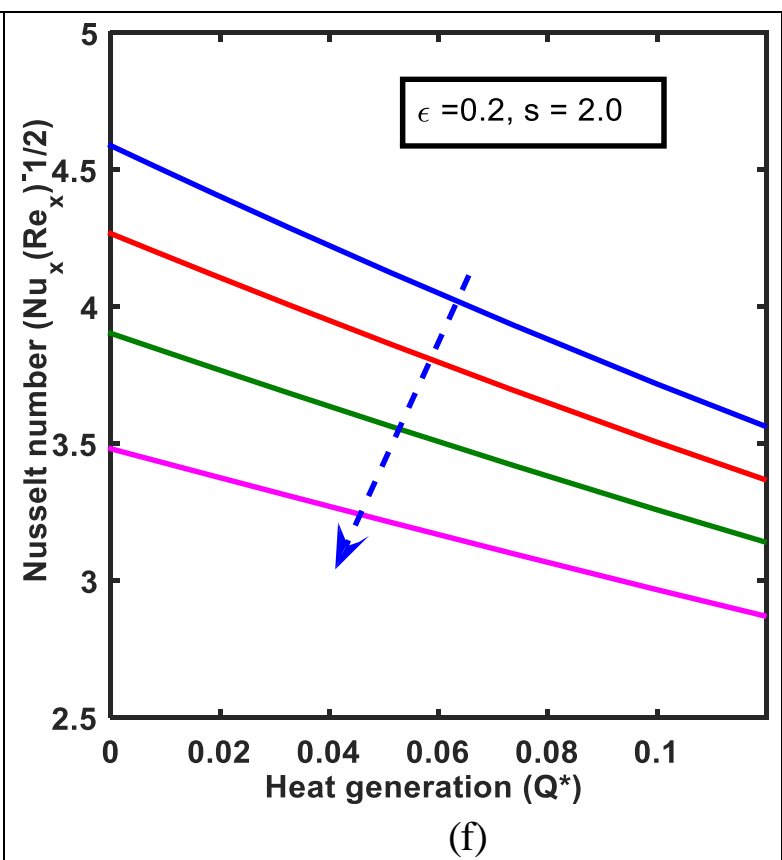

(f)

The graph of temperature profile represents that the fluid temperature within the boundary layer region is getting lower due to Prandtl number, power-law index parameter, and stretching ratio parameter whereas surface thickness parameter has a reverse effect on it throughout the boundary layer region. From these figures, it is also revealed that in the case of a thicker bullet-shaped object $(s=2.0)$ the temperature profile does not approach the ambient condition asymptotically but intersects the axis with a steep angle and the boundary layer structure has no definite shape whereas in the case of a thinner bullet-shaped object $(s=0.05)$ the temperature profile converge the ambient condition asymptotically and the boundary layer structure has a definite shape. Here also one can notice that the thinner bullet-shaped object acts as a good cooling conductor compared to the thicker bullet-shaped object for both $\epsilon=0.0$ and 2.0. It is also mentioned that the thinner bullet-shaped object represents a thinner thermal boundary layer because the heat transfer rate is higher than the thicker bullet-shaped object in both static and moving bullet-shaped objects. Hence it is mentioned that the lower values of the surface thickness parameter have a greater depressing effect on the temperature profile than higher values of the surface thickness parameter. It is also noticed that the thermal boundary layer thickness is higher for air when compared with water.

The variation of the Nusselt number against the effect of the Prandtl number displays in Figures $13 \mathrm{a}$ and $13 \mathrm{~b}$ by taking the stretching ratio parameter $\epsilon=0.0$ and $\epsilon=2.0$, and the surface thickness parameter $s=0.05$, respectively. 
Figure 13. Nusselt Number for Different Values of Prandtl Number when (a) $\epsilon=$ $0.0, s=0.05$, and $(b) \epsilon=2.0, s=0.05$

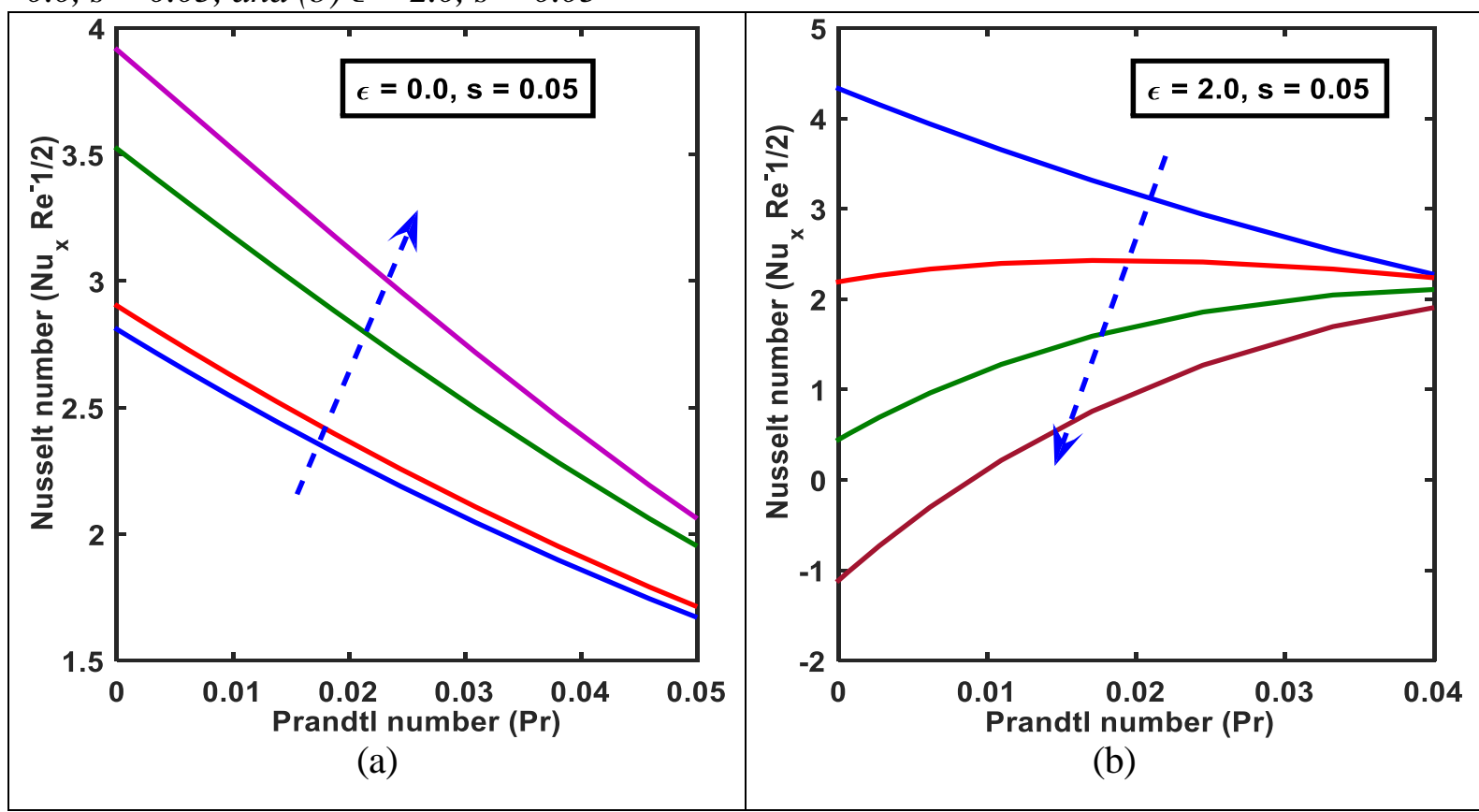

From these figures, it reveals that, the Nusselt number increase as the Prandtl number increases. It is observed that the Nusselt number is directly proportional to the temperature gradient. The temperature gradient at the surface is always negative for all values of the controlling parameters. Therefore, the Prandtl number is an increasing function of the Nusselt number. It is observed from Table 2 that, when Pr changes from 0.71 to 10.0 for $s=0.05$ and $\epsilon=0.0$, the Nusselt number increases $17.8 \%$ whereas for $s=0.05$ and $\epsilon=2.0$ the corresponding increases $48.8 \%$. Hence, the Nusselt number is higher for the stretching surface $(\epsilon=2.0)$ than the non-stretching surface $(\varepsilon=0)$.

\section{Influence of Power-Law Index Parameter (m) on Nusselt Number}

Figures $14 \mathrm{a}$ and $14 \mathrm{~b}$ represent the variation of Nusselt number with respect to power-law index parameter by taking the stretching ratio parameter $\epsilon=0.0$ and $\epsilon=$ 2.0, and the surface thickness parameter $s=0.05$, respectively. From these figures, it reveals that as the values of $m$ increases the Nusselt number also increases. Therefore, the power-law index parameter is directly proportional to the Nusselt number. 
Figure 14. Nusselt Number for Different Values of Power-Law Index Parameter (m) when $(a) \epsilon=0.0, s=0.05$, and $(b) \epsilon=0.0, s=2.0$

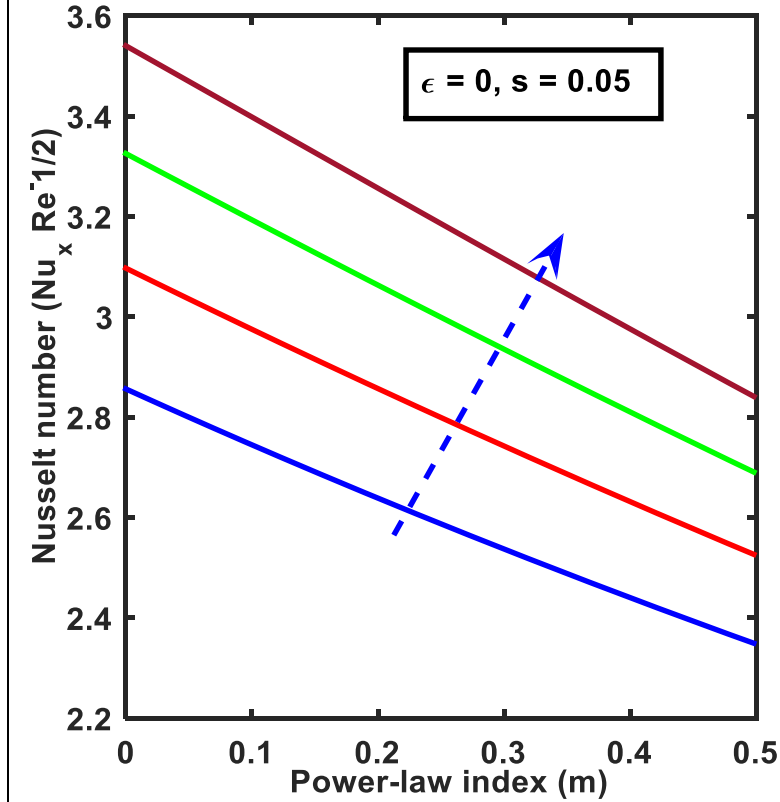

(a)

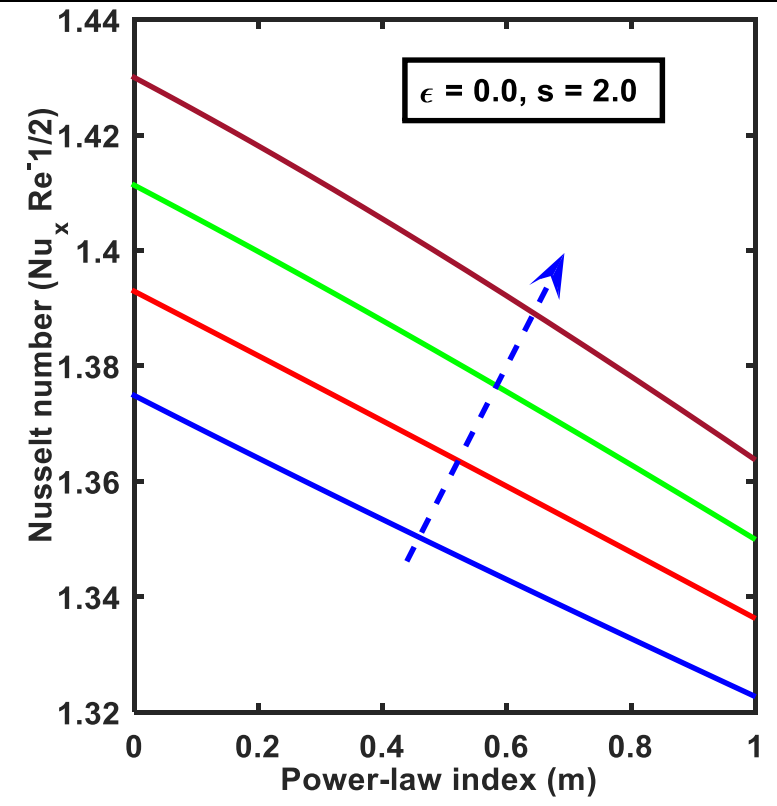

(b)

It is observed from Table 2 that, when $m$ changes from 0 to 2 for $s=0.05$ and $\epsilon=0.0$, the Nusselt number increases $8.85 \%$ whereas for $s=0.05$ and $\epsilon=2.0$ the corresponding increases $23.8 \%$. Hence, the Nusselt number is higher for the stretching surface $(\epsilon=2.0)$ than the non-stretching surface $(\varepsilon=0)$.

\section{Effect of Surface Thickness Parameter (s) on Nusselt Number}

Figures $15 \mathrm{a}$ and $15 \mathrm{~b}$ represent the variation of Nusselt number with respect to surface thickness parameter $(s)$ also taking the stretching ratio parameter $\epsilon=0.0$ and $\epsilon=2.0$, respectively. From these figures, it reveals that as the values of $s$ increase the Nusselt number decreases. Therefore, the Nusselt number is a decreasing function of the surface thickness parameter. This implies heat transfer rate is higher in the case of a thinner $(s=0.05)$ bullet-shaped object than the thicker $(s=2.0)$ bullet-shaped object. It is observed from Table 2 that, when $s$ changes from 0.05 to 0.3 for $\epsilon=0.0$, the Nusselt number decreases $85.76 \%$ whereas for $\epsilon=2.0$ the corresponding decreases $77.4 \%$. Hence, the Nusselt number is higher for $\epsilon=0.0$ than $\epsilon=2.0$. 
Figure 15. Nusselt Number for Different Values of Surface Thickness Parameter (s) when $(a) \epsilon=0.0$, and $(b) \epsilon=2.0$

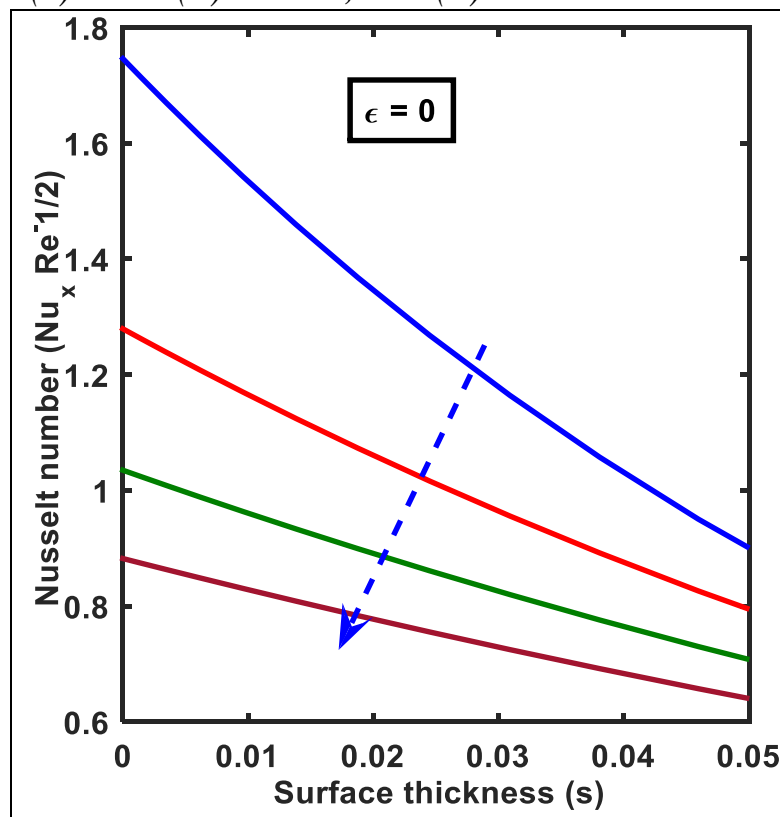

(a)

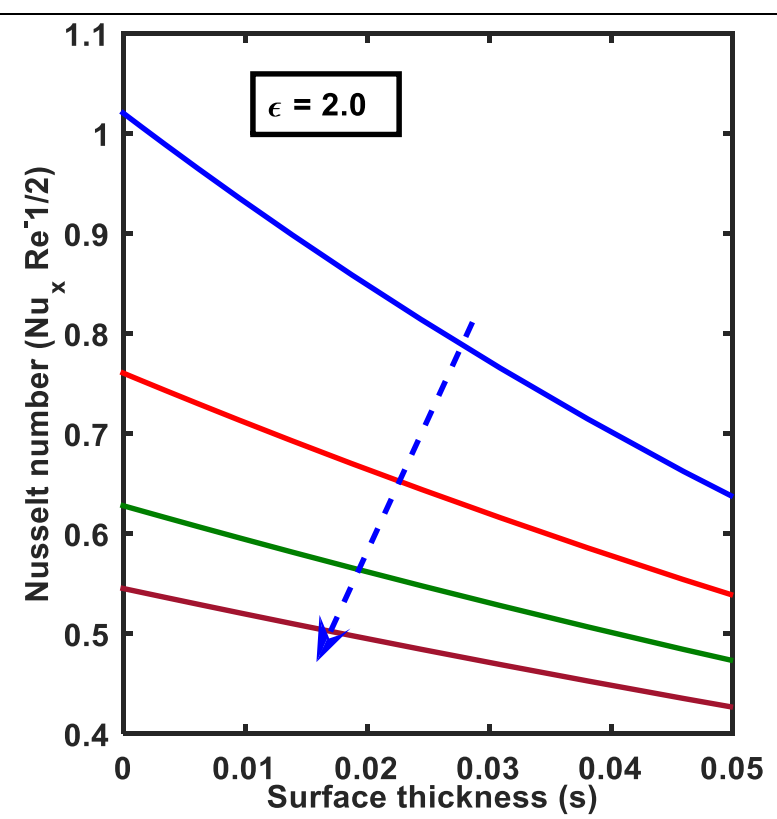

(b)

Table 1 represents the variation in skin friction coefficient for different values of the magnetic parameter $(M)$, surface thickness parameter $(s)$, Prandtl number $(\mathrm{Pr})$, and power-law index parameter $(\mathrm{m})$ for various movements of the bulletshaped object.

Table 1. Variation in Skin Friction Coefficient for Distinct Values of $M, m$, $s$, and Pr with $\epsilon=0.0,0.2,2.0, s=0.05$

\begin{tabular}{|c|c|c|c|c|c|c|}
\hline \multicolumn{4}{|c|}{ Parameters } & \multirow{2}{*}{$\begin{array}{l}-C_{f} \sqrt{\mathrm{Re}_{x}} \\
\epsilon=0.0, s=0.05\end{array}$} & \multirow{2}{*}{$\begin{array}{l}-C_{f} \sqrt{\mathrm{Re}_{x}} \\
\epsilon=0.2, s=0.05\end{array}$} & \multirow{2}{*}{$\begin{array}{c}\left\|-C_{f} \sqrt{\operatorname{Re}_{x}}\right\| \epsilon= \\
2.0, s=0.05\end{array}$} \\
\hline$M$ & $m$ & $s$ & $\operatorname{Pr}$ & & & \\
\hline 1.0 & 1.0 & 0.05 & 1.0 & 5.6404 & 4.5437 & 6.0131 \\
\hline 5.0 & 1.0 & 0.05 & 1.0 & 6.3441 & 5.1011 & 6.6555 \\
\hline 10.0 & 1.0 & 0.05 & 1.0 & 7.0787 & 5.6847 & 7.3436 \\
\hline 1.0 & 0.0 & 0.05 & 1.0 & 5.3397 & 4.2886 & 5.5421 \\
\hline 1.0 & 1.0 & 0.05 & 1.0 & 5.6404 & 4.5437 & 6.0131 \\
\hline 1.0 & 2.0 & 0.05 & 1.0 & 5.9128 & 4.7745 & 6.4342 \\
\hline 1.0 & 1.0 & 0.05 & 1.0 & 5.6404 & 4.5437 & 6.0131 \\
\hline 1.0 & 1.0 & 0.20 & 1.0 & 1.7491 & 1.4322 & 1.9969 \\
\hline 1.0 & 1.0 & 0.3 & 1.0 & 1.3251 & 1.0771 & 1.4993 \\
\hline 1.0 & 1.0 & 0.05 & 0.71 & 5.6404 & 4.5437 & 6.0131 \\
\hline 1.0 & 1.0 & 0.05 & 1.0 & 5.6404 & 4.5437 & 6.0131 \\
\hline 1.0 & 1.0 & 0.05 & 7.0 & 5.6404 & 4.5437 & 6.0131 \\
\hline
\end{tabular}

It is observed that the skin friction coefficient is inversely proportional to the velocity gradient, $f^{\prime \prime}(\eta)$ at the bullet-shaped object. So, the velocity gradient at the surface of the object is negative for all values of mentioned parameters in the case of $\varepsilon>1$ but positive when $\varepsilon<1$. The negative values $f^{\prime \prime}(\eta)$ mean that the bullet-shaped object exerts a drag force on the fluid flow whereas the positive 
values $f^{\prime \prime}(\eta)$ mean that the fluid flow exerts a drag force on the bullet-shaped object. From Table 1, it is observed that the magnetic parameter $(M)$ and powerlaw index parameter $(\mathrm{m})$ reducing the friction factor coefficient in the case of $\varepsilon<1$ but boosting the friction factor in the case of $\varepsilon>1$. On the other hand, the surface thickness parameter reduces the friction factor in both of the mentioned cases. It is interesting to note that friction factor coefficient is less in $\epsilon=0.2$ case when compared with $\epsilon=0.0, \epsilon=2.0$ cases. At this point, it is highlighted that for reducing the friction between the particles and surface we have to use $\epsilon=0.2$ cases. It is evident from this table that the skin friction coefficient is higher in $\epsilon=$ 2.0 case when comparing with $\epsilon=0.0, \epsilon=0.2$ cases. It is also noticed that the Prandtl number (Pr) does not affect the skin friction coefficient.

Table 2 displays the variation in Nusselt number for different values of the magnetic parameter $(M)$, surface thickness parameter $(s)$, Prandtl number (Pr), and power-law index parameter $(m)$ for various movements of the bullet-shaped object. It is observed that the Nusselt number is directly proportional to the heat transfer rate $\theta^{\prime}(\eta)$ at the bullet-shaped object. The heat transfer rate at the surface of the object $\theta^{\prime}(\eta)$ is negative for all values of mentioned parameters. From Table 2 , it is possible to observe that the heat transfer rate increases for the magnetic parameter $(M)$, Prandtl number $(\mathrm{Pr})$, and power-law index parameter $(m)$ but decreases for the surface thickness parameter $(s)$ for all three cases of stretching ratio parameter $(\epsilon=0.0, \epsilon=0.2, \epsilon=2.0)$ and $\mathrm{s}=0.05$ respectively. On the other hand, in the case of $\epsilon=2.0$, and $\mathrm{s}=0.05$ the heat transfer rate increases for the magnetic parameter $(M)$ and surface thickness parameter $(s)$ whereas decreases for Prandtl number $(\operatorname{Pr})$, and power-law index parameter $(m)$. Therefore, heat transfer rate is high in $\epsilon=0.0$ case, when compared with $\epsilon=0.2, \epsilon=2.0$ cases. At this point, it is highlighted that for reducing the heat transfer rate we have to consider the static $(\epsilon=0.0)$ bullet-shaped object.

Table 2. Variation in Nusselt Number, $N u_{x}\left(\sqrt{\operatorname{Re}_{x}}\right)^{-1}$ for Different Values of $M$, $m, s$, and $\operatorname{Pr}$ with $\epsilon=0.0,0.2,2.0, s=0.05$

\begin{tabular}{|c|c|c|c|c|c|c|}
\hline \multicolumn{4}{|c|}{ Parameters } & \multirow{3}{*}{$\begin{array}{c}\|-N u_{x}\left(\sqrt{\mathrm{Re}_{x}}\right)^{-1} \\
\epsilon=0.0, \mathrm{~s}=0.05\end{array}$} & \multirow{3}{*}{$\begin{array}{c}\left\|-N u_{x}\left(\sqrt{\operatorname{Re}_{x}}\right)^{-1}\right\| \\
\epsilon=0.2, \mathrm{~s}=0.05\end{array}$} & \multirow{3}{*}{$\begin{array}{c}\left\|-N u_{x}\left(\sqrt{\operatorname{Re}_{x}}\right)^{-1}\right\| \\
\epsilon=2.0, \mathrm{~s}=0.05\end{array}$} \\
\hline $\mathrm{M}$ & $\mathrm{m}$ & $\mathrm{s}$ & $\operatorname{Pr}$ & & & \\
\hline & & & & & & \\
\hline 1.0 & 1.0 & 0.05 & 1.0 & 10.4671 & 10.6800 & 11.2800 \\
\hline 5.0 & 1.0 & 0.05 & 1.0 & 10.4911 & 10.6912 & 11.2611 \\
\hline 10.0 & 1.0 & 0.05 & 1.0 & 10.5134 & 10.7019 & 11.2426 \\
\hline 1.0 & 0.0 & 0.05 & 1.0 & 10.0001 & 10.0001 & 10.0000 \\
\hline 1.0 & 1.0 & 0.05 & 1.0 & 10.4671 & 10.6800 & 11.2800 \\
\hline 1.0 & 2.0 & 0.05 & 1.0 & 10.8845 & 11.2824 & 12.3826 \\
\hline 1.0 & 1.0 & 0.05 & 1.0 & 10.4671 & 10.6800 & 11.2800 \\
\hline 1.0 & 1.0 & 0.20 & 1.0 & 2.9202 & 3.1327 & 3.4985 \\
\hline 1.0 & 1.0 & 0.30 & 1.0 & 1.4899 & 2.3417 & 2.5867 \\
\hline 1.0 & 1.0 & 0.05 & 0.71 & 10.3431 & 10.4981 & 10.9376 \\
\hline 1.0 & 1.0 & 0.05 & 1.0 & 10.4671 & 10.6800 & 11.2800 \\
\hline 1.0 & 1.0 & 0.05 & 7.0 & 12.1862 & 13.3201 & 16.2761 \\
\hline
\end{tabular}


Figures $16 \mathrm{a}$ and $16 \mathrm{~b}$ show the convergence and accuracy of the present problem. Figure 16a depicts the infinity norms with iterations. The error infinity norm decreases with the increasing number of iterations that confirms the convergence of the present method. So, the present method converges after five iterations. Figure $16 \mathrm{~b}$ represents the residual error norms of less than $10^{-8}$ and $10^{-14}$ for $f(\eta)$ and $\theta(\eta)$ against after fourth iterations. It is seen that the residual error decreases with increasing the iterations. This proves the validity of the present method. The errors show that the SQLM is accurate giving errors of less than $10^{-8}$ within the fourth iteration.

Figure 16. (a) Error Infinity Norms and (b) Residual Error Infinity Norms for $f(\eta)$ and $\theta(\eta)$

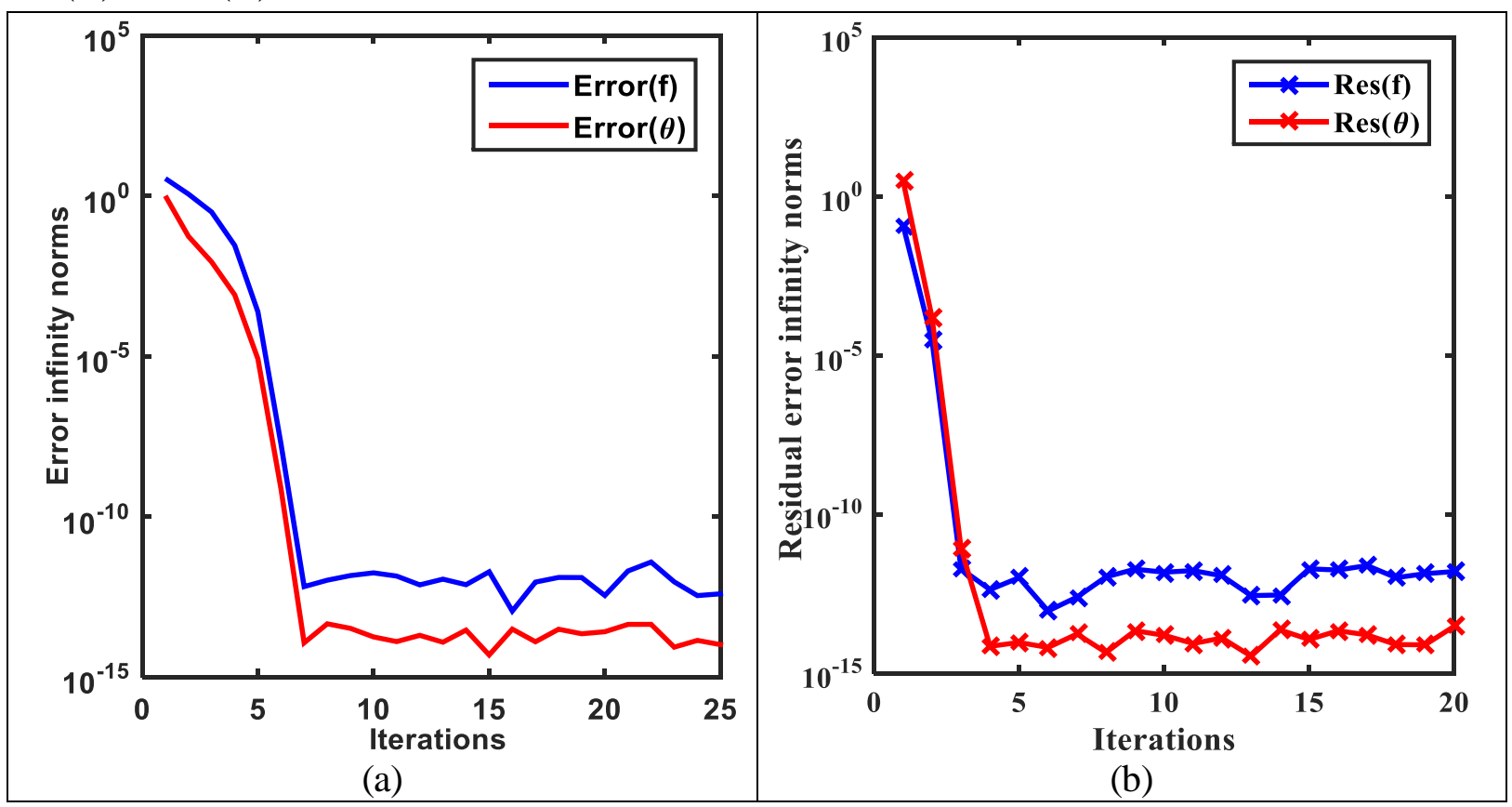

\section{Correlation Analysis for Velocity Gradient}

A positive correlation means higher values of one variable tend to higher values of another variable but the reverse case arises for the negative correlation. The statistically significant values of the parameters are highlighted in the table because the values are greater equal to 0.25 . Then we say that a $95 \%$ chance of a relationship between the parameters. So, from Table 3 it is observed that the velocity gradient is positively correlated with the magnetic parameter $(M)$, and power-law index parameter $(m)$ but negatively correlated with the surface thickness parameter $(s)$ and stretching ratio parameter $(\epsilon)$. Therefore, the fluid velocity and gradient of velocity is an increasing function of the magnetic parameter, and power-law index parameter but $a$ decreasing function of the surface thickness parameter and stretching ratio parameter within the boundary layer region. 
Table 3. Correlation Coefficient between the Velocity Gradient $f^{\prime \prime}(\eta)$ and Controlling Parameters by Taking $1^{\text {st }}$ Order Coefficient in the Case of $\epsilon=0.2$

\begin{tabular}{|l|c|c|c|c|c|}
\hline Parameters & $M$ & $s$ & $m$ & $\epsilon$ & $f^{\prime \prime}(\eta)$ \\
\hline$M$ & 1.00 & & & & \\
\hline$s$ & -0.22 & 1.00 & & & \\
\hline$m$ & -0.09 & -0.08 & 1.00 & & \\
\hline$\epsilon$ & 0.17 & 0.15 & 0.07 & 1.00 & \\
\hline$f^{\prime \prime}(0)$ & $\mathbf{0 . 5 2}$ & $\mathbf{- 0 . 4 3}$ & $\mathbf{0 . 2 1}$ & $\mathbf{- 0 . 3 3}$ & 1.00 \\
\hline
\end{tabular}

\section{Correlation Analysis for Temperature Gradient}

A positive correlation means higher values of one variable tend to higher values of another variable but the reverse case arises for the positive correlation. The correlations are significant between the variables if the numerical value of 0.25 or above. Then we say that a $95 \%$ chance of a relationship between the parameters. So, from Table 4 the correlation is significant for all mentioned parameters except the stretching ratio parameter which is highlighted in the table. The temperature gradient is positively correlated with the parameters $\operatorname{Pr}, \mathrm{M}, \mathrm{m}$, and $\epsilon$ but negatively correlated with the parameter $s$ and $Q^{*}$.

Table 4. Correlation Coefficient Between the Temperature Gradient $\theta^{\prime}(\eta)$ and Controlling Parameters by Taking Zero-Order Coefficient

\begin{tabular}{|c|c|c|c|c|c|c|c|}
\hline \multicolumn{7}{|c|}{ Correlation Summary } & \\
\hline Parameters & $\operatorname{Pr}$ & $M$ & $m$ & $s$ & $Q^{*}$ & $\epsilon$ & $\theta^{\prime}(\eta)$ \\
\hline $\operatorname{Pr}$ & 1.00 & & & & & & \\
\hline$M$ & 0.59 & 1.00 & & & & & \\
\hline$m$ & -0.08 & -0.13 & 1.00 & & & & \\
\hline$s$ & -0.14 & -0.21 & -0.08 & 1.00 & & & \\
\hline$Q^{*}$ & -0.09 & -0.06 & -0.09 & 0.00 & $1 . .00$ & & \\
\hline$\epsilon$ & 0.00 & 0.00 & 0.00 & 0.00 & -0.09 & 1.00 & \\
\hline$\theta^{\prime}(\eta)$ & $\mathbf{0 . 4 5}$ & $\mathbf{0 . 4 4}$ & $\mathbf{0 . 2 8}$ & $\mathbf{- 0 . 6 6}$ & -0.15 & $\mathbf{0 . 2 3}$ & 1.00 \\
\hline
\end{tabular}

From Table 5 it is observed that all the parameters are statistically significant because their P - VALUEs less than 0.05 . Hence at least a $95 \%$ chance that there is a true relationship between the parameters and the velocity gradient. From the regression model it is observed that if we increase the one-unit value of the magnetic parameter $(\mathrm{M})$ and power - law index parameter $(\mathrm{m})$ then the average decrease of the skin friction coefficient by 0.8 . From the table the regression model is

$$
f^{\prime \prime}(\eta)=0.2 M+1.1 m-0.6 s+1.3 \operatorname{Pr}-0.3 \varepsilon
$$


Table 5. Regression Analyses for the Dimensionless Parameters $M, m$, and $s$ and the Velocity $f^{\prime \prime}(\eta)$ by Taking $2^{\text {nd }}$ Order Coefficient

\begin{tabular}{|l|c|c|c|c|c|c|}
\hline \multicolumn{7}{|c|}{ Regression Statistics } \\
\hline Multiple R & 0.758 & & & & & \\
\hline R Square & 0.575 & & & & & \\
\hline $\begin{array}{l}\text { Adjusted R } \\
\text { Square }\end{array}$ & 0.368 & & & & & \\
\hline Standard Error & 1.368 & & & & & \\
\hline Observations & 18.00 & & & & & \\
\hline & df & SS & MS & F & Significance F & \\
\hline Regression & 5.00 & 32.946 & 6.589 & 3.522 & 0.034 & \\
\hline Residual & 13.00 & 24.321 & 1.871 & & & \\
\hline Total & 18.00 & 57.267 & & & & \\
\hline & Coefficients & Standard & t Stat & P-value & Lower $95 \%$ & Upper \\
& & Error & & & & \#5\% \\
\hline Intercept & 0.00 & \#N/A & \#N/A & \#N/A & \#N/A & \#/A \\
\hline$M$ & 0.2 & 0.064 & -3.039 & $\mathbf{0 . 0 0 9}$ & -0.335 & -0.057 \\
\hline$s$ & -0.6 & 0.687 & -0.866 & 0.402 & -2.080 & 0.889 \\
\hline$m$ & 1.1 & 0.426 & -2.366 & $\mathbf{0 . 0 3 4}$ & -1.929 & -0.088 \\
\hline Pr & 1.3 & 0.803 & 1.569 & 0.141 & -0.475 & 2.993 \\
\hline$\epsilon$ & -0.3 & 0.427 & -0.673 & 0.513 & -1.210 & 0.636 \\
\hline
\end{tabular}

From Table 6 the regression model is $\theta^{\prime}(\eta)=1.841+0.19 \operatorname{Pr}+0.16 M+0.47 m-1.21 s+0.33 \varepsilon-0.3 Q^{*}$. In the regression analysis the variables are significant if $\mathrm{P}-\mathrm{VALUE}$ less than 0.05 and this is the condition to develop a true relationship between dependent and independent variables. From Table 6 the significant variables are only the surface thickness parameter (s) which is highlighted in the table. From the regression model it is observed that if we increase the one unit value of the Prandtl number $(\operatorname{Pr})$, power law index parameter $\mathrm{m}$, surface thickness parameter $\mathrm{s}$ and stretching ratio $(\epsilon)$ and then the average increase of the skin friction coefficient by $0.307,0.0548,2.13$ and 0.853. Again, if we increase the one-unit value of surface thickness parameter $\mathrm{s}$ then the average decreases of the skin friction coefficient by 21.71 .

Table 6. Regression Analyses of the Energy Transfer $\theta^{\prime}(\eta)$ by Taking 0 (Zero) Order Coefficient

\begin{tabular}{|l|c|c|c|c|c|c|}
\hline \multicolumn{7}{|c|}{ Regression Statistics } \\
\hline Multiple R & 0.841 & & & & & \\
\hline R Square & 0.708 & & & & & \\
\hline $\begin{array}{l}\text { Adjusted R } \\
\text { Square }\end{array}$ & 0.525 & & & & & \\
\hline Standard Error & 0.773 & & & & & \\
\hline Observations & 14.00 & & & & & \\
\hline & $\mathrm{df}$ & SS & MS & F & Significance F & \\
\hline Regression & 5.00 & 11.56 & 2.312 & 3.873 & $\mathbf{0 . 0 4 4}$ & \\
\hline Residual & 8.00 & 4.776 & 0.597 & & & \\
\hline Total & 13.00 & 16.36 & & & & \\
\hline & Coefficients & Standard Error & t Stat & P-value & Lower 95\% & Upper $95 \%$ \\
\hline
\end{tabular}




\begin{tabular}{|l|c|c|c|c|c|c|} 
Intercept & 1.841 & 0.602 & 3.05 & 0.016 & 0.452 & 3.230 \\
\hline $\mathrm{Pr}$ & 0.184 & 0.152 & 1.21 & 0.260 & -0.166 & 0.535 \\
\hline $\mathrm{M}$ & 0.162 & 0.204 & 0.79 & 0.450 & -0.309 & 0.633 \\
\hline $\mathrm{m}$ & 0.469 & 0.328 & 1.43 & 0.190 & -0.287 & 1.226 \\
\hline $\mathrm{s}$ & -1.213 & 0.432 & -2.80 & $\mathbf{0 . 0 2 3}$ & -2.208 & -0.217 \\
\hline $\mathrm{Q}^{*}$ & -0.30 & 0.07 & -3.96 & 0.00 & -0.45 & -0.14 \\
\hline$\epsilon$ & 0.325 & 0.273 & 1.19 & 0.26 & -0.305 & 0.955 \\
\hline
\end{tabular}

\section{Code Verification}

The numerical values $f^{\prime \prime}(\eta)$ have been compared with the results of Afridi and Qasim (2018) for different values of $\eta$ to validate the convergence and accuracy of the present method. The results are almost similar to the previous results which are shown in Table 7.

Table 7. Comparison of Skin Friction $f^{\prime \prime}(\eta)$ for Different Values of $\eta$ with Afridi (2018) by Taking $M=m=0$ and $\operatorname{Pr}=1$

\begin{tabular}{|l|c|c|}
\hline & Afridi & Present results \\
\hline$\eta$ & $f^{\prime \prime}(0)$ & $f^{\prime \prime}(\eta)$ \\
\hline 0.001 & $\mathbf{6 2 . 1 6 3 7}$ & $\mathbf{6 2 . 1 5 7 2}$ \\
\hline 0.01 & $\mathbf{8 . 4 9 2 4}$ & $\mathbf{8 . 4 9 1 2}$ \\
\hline 0.10 & $\mathbf{1 . 2 8 8 8}$ & $\mathbf{1 . 2 8 3 9}$ \\
\hline 0.15 & - & $\mathbf{0 . 9 3 5 9}$ \\
\hline
\end{tabular}

\section{Conclusions}

The effect of various parameters on MHD laminar boundary layer flow of an incompressible, electrically conducting, and viscous Newtonian fluid past a stretching electrically non-conducting bullet-shaped object with heat transfer has been carried out. In sequence, the fluid flow and heat transfer have been investigated in two types of flow geometries such as the thicker surface $(s \geq 2)$ and the thinner surface $(0<s<2)$ of the bullet-shaped object.

- The SQLM along with the Chebyshev collocation method provides a more accurate and quicker convergence scheme.

- Velocity profile squeezes for the limit $\varepsilon>1$ but in the limit, $\varepsilon<1$ the velocity profile enhances for increasing the shape and size (surface thickness parameter, $s$ ) of the bullet-shaped object but the boundary layer thickness expands in both cases.

- In the case of the thinner bullet-shaped object, $(0<s<2)$ the fluid velocity profile converges asymptotically to the free stream velocity at infinity whereas for a thicker bullet-shaped object $(s \geq 2)$ boundary conditions do not satisfy. 
- A thin momentum boundary layer thickness has been found in a thinner bullet-shaped object $(0<s<2)$ than the thicker bullet-shaped object $(s \geq 2)$.

- The thinner bullet-shaped object $(0<s<2)$ represents a thinner thermal boundary layer because the heat transfer rate is higher than the thicker bullet-shaped object $(s \geq 2)$.

- There is a positive correlation exists of the magnetic parameter (M) and power - law index $(\mathrm{m})$ with velocity gradient and but negative correlation exists for surface thickness parameter (s) whereas there is no correlation for Prandtl number (Pr).

- A strong positive correlation exists with temperature gradient of the parameters such as Prandtl number, power - law index whereas a negative correlation exists of the magnetic, heat generation and surface thickness parameters. Further, a positive weak correlation exists between temperature gradient and stretching ratio parameter.

- The skin friction reduces for the magnetic parameter and power - law index parameter but increases for surface thickness parameter rate and stretching ratio parameter.

\section{References}

Abo-Eldahab EM, Salem AM (2004) Hall effects on MHD free convection flow of a nonNewtonian power-law fluid at a stretching surface. International Communications in Heat and Mass Transfer 31(3): 343-354.

Afridi IM, Qasim M (2018) Entropy generation and heat transfer in boundary layer flow over a thin needle moving in a parallel stream in the presence of nonlinear Rosseland radiation. International Journal of Thermal Sciences 123(Jan): 117-128.

Aftab A, Javed IS, Muhammad S (2018) Dual solutions in a boundary layer flow of a power-law fluid over a moving permeable flat plate with thermal radiation, viscous dissipation and heat generation/absorption. Fluids 3(1): 1-6.

Ahmad B, Nieto J, Shahzad N (2001) The Bellman quasi-linearization method for Neumann problems. Journal of Mathematical Analysis and Applications 257(2): 356363.

Ahmad B, Nieto J, Shahzad N (2002) Generalized quasi-linearization method for mixed boundary value problems. Applied Mathematics and Computation 133(2-3): 423-429.

Ajala OA, Aselebe LO, Abimbade SF, Ogunsola AW (2019) Effect of magnetic fields on the boundary layer flow of heat transfer with variable viscosity in the presence of thermal radiation. International Journal of Scientific and Research Publication 9(5): 13-19.

Ajaykumar M, Srinivasa AH (2020) Variable viscosity effects on unsteady MHD laminar boundary layer flow and heat transfer over a stretching sheet. In AIP Conference Proceedings, 2277.

Alam M S, Asiya KM, Rahman MM, Vajravelu K (2016) Effects of variable fluid properties and thermophoresis on unsteady forced convective boundary layer flow 
along a permeable stretching/shrinking wedge with variable Prandtl and Schmidt numbers. International Journal of Mechanical Sciences 105(Jan): 191-205.

Alarifi IM, Ahmed GA, Osman M, Liaquat AL, Ayed MB, Belmabrouk H, et al. (2019) MHD flow and heat transfer over a vertical stretching sheet with heat sink or source effect. Symmetry 11(3): 297.

Asaithambi A (1998) A finite-difference method for the solution of the Falkner-Skan equation. Applied Mathematics and Computation 92(2-3): 135-141.

Ashwini G, Eswara AT (2012) MHD Falkner-Skan boundary layer flow with internal heat generation or absorption. International Journal of Mathematical, Computational, Physical, Electrical and Computer Engineering 6(5): 556-559.

Ashwini G, Eswara AT (2015) Unsteady MHD decelerating flow over a wedge with heat generation/absorption. International Journal of Mathematics and Computer Science 1(5): 303-309.

Awaludin IS, Ishak A, Pop I (2018) On the stability of MHD boundary layer flow over a stretching/shrinking wedge. Scientific Reports 8(1): 13622.

Bellman R E, Kalaba R E (1965) Quasilinearization and nonlinear boundary value problems. New York, USA: American Elsevier.

Daba M, Devaraj P, Subhashini SV (2015) Mixed convection boundary layer flow over a vertical stretching sheet with convective boundary condition and effect of partial slip. Frontiers in Heat and Mass Transfer 6(1): 1-6.

Falana A, Ojewale OA, Adeboje TB (2016) Effect of Brownian motion and thermophoresis on a nonlinearly stretching permeable sheet in a nanofluid. Advances in Nanoparticles 5(1): 140-151.

Ibrahim W, Tulu A (2019) Magnetohydrodynamic (MHD) boundary layer flow past a wedge with heat transfer and viscous effects of nanofluid embedded in porous media. Mathematical Problems in Engineering 1(Jan): 1-12.

Irfan M, Farooq MA, Mushtaq A, Shamsi ZH (2020) Unsteady MHD bio-nanofluid flow in a porous medium with thermal radiation near a stretching/shrinking sheet. Mathematical Problems in Engineering (special issue): 8822999.

Jabeen K, Mushtaq M, Akram R M (2020) Analysis of the MHD bboundary layer flow over a nonlinear sstretching sheet in a porous medium using semianalytical approaches. Mathematical Problems in Engineering 5(Feb): 3012854.

Kandasamy R, Mohammad R (2015) Radiative heat transfer on nanofluids flow over a porous convective surface in the presence of magnetic field. Journal of Applied Mechanical Engineering 4(4): 1-7.

Kumar P, Krishnan K (2018) Analysis of axisymmetric boundary layers. Journal of Fluid Mechanics 849(Jun): 927-941.

Mabood F, Pochai N, Shateyi S (2016) Stagnation point flow of nanofluid over a moving plate with convective boundary condition and magnetohydrodynamics. Journal of Engineering (4): 5874864.

Marneni N, Ashraf M, (2015) Mixed convection flow over a permeable stretching wedge in the presence of heat generation/absorption, viscous dissipation, radiation and ohmic heating. Chemical Engineering Transactions 45(Oct): 955-960.

Megahed AM, Reddy GM, Abbas W (2021) Modeling of MHD fluid flow over an unsteady stretching sheet with thermal radiation, variable fluid properties and heat flux. Mathematics and Computers in Simulation 185(Jan): 583-593.

Mustaqim MJ, Fadzilah MA, Arifin MA, Bachok N (2018) A stability analysis of stagnation-point flow of heat and mass transfer over a shrinking sheet with radiation and slip effects. International Journal of Advanced Science Engineering and Technology 6(Mar): 2321-8991. 
Muthukumaran C, Bathrinathan K (2020) Mathematical modeling of mixed convection boundary layer flows over a stretching sheet with viscous dissipation in presence of suction and injection. Symmetry 12(11): 1754.

Nageeb A, Haroun H, Mondal S, Sibanda P (2017) Effects of thermal radiation of mixed convection in a MHD nanofluid flow over a stretching sheet using a spectral relaxation method. International Journal of Mathematical and Computational Sciences 11(2): 1-10.

Salleh SNA, Bachok N, Arifin MN, Ali MF (2019) Numerical analysis of boundary layer flow adjacent to a thin needle in nanofluid with the presence of heat source and chemical reaction. Symmetry 11(4): 543.

Shateyi S, Muzara H (2020) On numerical analysis of Carreau-Yasuda nanofluid flow over a non-linearly stretching sheet under viscous dissipation and chemical reaction effects. Computation 8(7): 1148. 
